\title{
Neural dynamics of brightness perception: Features, boundaries, diffusion, and resonance
}

\author{
MICHAEL A. COHEN and STEPHEN GROSSBERG \\ Boston University, Boston, Massachusetts
}

\begin{abstract}
A real-time visual processing theory is used to unify the explanation of monocular and binocular brightness data. This theory describes adaptive processes which overcome limitations of the visual uptake process to synthesize informative visual representations of the external world. The brightness data include versions of the Craik-O'Brien-Cornsweet effect and its exceptions, Bergström's demonstrations comparing the brightnesses of smoothly modulated and step-like luminance profiles, Hamada's demonstrations of nonclassical differences between the perception of luminance decrements and increments, Fechner's paradox, binocular brightness averaging, binocular brightness summation, binocular rivalry, and fading of stabilized images and ganzfelds. Familiar concepts such as spatial frequency analysis, Mach bands, and edge contrast are relevant but insufficient to explain the totality of these data. Two parallel contour-sensitive processes interact to generate the theory's brightness, color, and form explanations. A boundary-contour process is sensitive to the orientation and amount of contrast but not to the direction of contrast in scenic edges. It generates contours that form the boundaries of monocular perceptual domains. The spatial patterning of these contours is sensitive to the global configuration of scenic elements. A featurecontour process is insensitive to the orientation of contrast, but is sensitive to both the amount of contrast and to the direction of contrast in scenic edges. It triggers a diffusive filling-in reaction of featural quality within perceptual domains whose boundaries are dynamically defined by boundary contours. The boundary-contour system is hypothesized to include the hypercolumns in visual striate cortex. The feature-contour system is hypothesized to include the blobs in visual striate cortex. These preprocessed monocular activity patterns enter consciousness in the theory via a process of resonant binocular matching that is capable of selectively lifting whole monocular patterns into a binocular representation of form-and-color-in-depth. This binocular process is hypothesized to occur in area V4 of the visual prestriate cortex.
\end{abstract}

\section{(1) Paradoxical Percepts as Probes of Adaptive Processes}

This article describes quantitative simulations of monocular and binocular brightness data to illustrate and support a real-time perceptual processing theory. This theory introduces new concepts and mechanisms concerning how human observers achieve informative perceptual representations of the external world that overcome limitations of the sensory uptake process, notably of how distributed patterns of locally ambiguous visual features can be used to generate unambiguous global percepts.

For example, light passes through retinal veins before it reaches retinal photoreceptors. Human observers do not perceive their retinal veins due to the action of mechanisms that attenuate the perception of images that are stabilized with respect to the retina. Mechanisms capable of

M. A. Cohen was supported in part by the National Science Foundation (NSF-IST-80-00257) and the Office of Naval Research (ONRN00014-83-K0337). S. Grossberg was supported in part by the Air Force Office of Scientific Research (AFOSR 82-0148) and the Office of Naval Research (ONR-N00014-83-K0337). We thank Cynthia Suchta for her valuable assistance in the preparation of the manuscript and illustrations.

The authors' mailing address is: Center for Adaptive Systems, Boston University, 111 Cummington Street, Boston, MA 02215. generating this adaptive property of visual percepts can also generate paradoxical percepts, as during the perception of stabilized images or ganzfelds (Pritchard, 1961; Pritchard, Heron, \& Hebb, 1960; Riggs, Ratliff, J. C. Cornsweet, \& T. N. Cornsweet, 1953; Yarbus, 1967). Once such paradoxical percepts are traced to an adaptive perceptual process, they can be used as probes to discover the rules governing this process. This type of approach has been used throughout the research program on perception (Carpenter \& Grossberg, 1981; Cohen \& Grossberg, 1984; Grossberg, 1980, 1982a, 1983a, 1983b; Grossberg \& Mingolla, 1985, in press) of which this work forms a part.

Suppressing the perception of stabilized veins is insufficient to generate an adequate percept. The images that reach the retina can be occluded and segmented by the veins in several places. Somehow, broken retinal contours need to be completed, and occluded retinal color and brightness signals need to be filled in. Holes in the retina, such as the blind spot or certain scotomas, are also not visually perceived (Gerrits, de Haan, \& Vendrick, 1966; Gerrits \& Timmermann, 1969; Gerrits \& Vendrick, 1970) due to some sort of filling-in process. These completed boundaries and filled-in colors are illusory percepts, 
albeit illusory percepts with an important adaptive value. The large literature on illusory figures and filling in can thus be used as probes of this adaptive process (Arend, Buehler, \& Lockhead, 1971; Day, 1983; Gellatly, 1980; Kanizsa, 1974; Kennedy, 1978, 1979, 1981; Parks, 1980; Parks \& Marks, 1983; Petry, Harbeck, Conway, \& Levey, 1983; Redies \& Spillmann, 1981; van Tuijl, 1975; van Tuijl \& de Weert, 1979; Yarbus, 1967). The brightness simulations that we report herein illustrate our theory's proposal for how real and illusory boundaries are completed and features are filled in.

Retinal veins and the blind spot are not the only blemishes of the retinal image. The luminances that reach the retina confound inhomogeneous lighting conditions with invariant object reflectances. Workers since the time of Helmholtz (Helmholt, 1962) have realized that the brain somehow "discounts the illuminant" to generate color and brightness percepts that are more accurate than the retinal data. Land (1977) has shown, for example, that the perceived colors within a picture constructed from overlapping colored patches are determined by the relative contrasts at the edges between the patches. The luminances within the patches are somehow discounted. These data also point to the existence of a filling-in process. Were it not possible to fill in colors to replace the discounted illuminants, we would perceive a world of boundaries rather than one of extended forms.

Since edges are used to generate filled-in percepts, an adequate perceptual theory must define edges in a way that can accomplish this goal. We suggest that the edge computations whereby boundaries are completed are fundamentally different-in particular, they obey different rules-from the edge computations leading to color and brightness signals. We claim that both types of edges are computed in parallel before being recombined to generate filled-in percepts. Our theory hereby suggests that the fundamental question "What is an edge, perceptually speaking?" has not adequately been answered by previous theories. One consequence of our answer is a physical explanation and generalization of the retinex theory (Grossberg, 1985), which Land (1977) has developed to explain his experiments.

The present article further supports this conception of how edges are computed by qualitatively explaining, and quantitatively simulating on the computer, such paradoxical brightness data as versions of the Craik-O'BrienCornsweet effect (Arend et al., 1971; Cornsweet, 1970; O'Brien, 1958) and its exceptions (Coren, 1983; Heggelund \& Krekling, 1976; van den Brink \& Keemink, 1976; Todorović, 1983), the Bergström demonstrations comparing the brightnesses of smoothly modulated and step-like luminance profiles (Bergström, 1966, 1967a, 1967b), and the demonstrations of Hamada (1980) showing nonclassical differences between the perception of luminance decrements and increments. These percepts can all be seen with one eye. Our theory links these phenomena to the visual mechanisms that are capable of preventing perception of retinal veins and the blind spot, and that fill in over discounted illuminants, which also operate when only one eye is open.

Due to the action of binocular visual mechanisms that generate a self-consistent percept of depthful forms, some visual images that can be monocularly perceived may not be perceived during binocular viewing conditions. Binocular rivalry provides a classical example of this fact (Blake \& Fox, 1974; Cogan, 1982; Kaufman, 1974; Kulikowski, 1978). To support the theory's conception of how depthful form percepts are generated (Cohen \& Grossberg, 1984; Grossberg, 1983a, 1983b), we suggest explanations and provide simulations of data concerning inherently binocular brightness interactions. These data include results on Fechner's paradox, binocular brightness summation, binocular brightness averaging, and binocular rivalry (Blake, Sloane, \& Fox, 1981; Cogan, 1982; Cogan, Silverman, \& Sekuler, 1982; Curtis \& Rule, 1980; Legge \& Rubin, 1981; Levelt, 1965).

These simulations do not, of course, begin to exhaust the richness of the perceptual literature. They are meant to be illustrative, rather than exhaustive, of a perceptual theory that is still undergoing development. On the other hand, this incomplete theory already reveals the perhaps even more serious incompleteness of rival theories by suggesting concepts and explaining data that are outside the range of these rival theories. The article also illustrates the theory's burgeoning capacity to integrate the explanation of perceptual data by providing simulations of data about Fechner's paradox, binocular brightness averaging, binocular brightness summation, and binocular rivalry using the same model parameters that were established to simulate disparity matching, filling-in, and figure-ground synthesis (Cohen \& Grossberg, 1984).

Although our theory was derived from perceptual data and concepts, after it reached a certain state in its development, striking formal similarities with recent neurophysiological data could not fail to be noticed. Some of these relationships are briefly summarized in Table 1 below. Although the perceptual theory can be understood without considering its neurophysiological interpretation, if one is willing to pursue this interpretation, then the perceptual theory implies a number of neurophysiological and anatomical predictions. Such predictions enable yet another data base to be used for the further development and possible disconfirmation of the theory.

A search through the neurophysiological literature has revealed that some of these predictions were already supported by known neural data, albeit data that took on new meaning in the light of the perceptual theory. Not all of the predictions were known, however. In fact, two of its predictions about the process of boundary completion have recently received experimental support from recordings by von der Heydt, Peterhans, and Baumgartner (1984) on cells in area 18 of the monkey visual cortex. Neurophysiological interpretations and predictions of the theory are described in Grossberg and Mingolla (in press). 
Due to the existence of this neural interpretation, we will take the liberty of calling the formal nodes in our network "cells" throughout the article. The next sections summarize the concepts that we use to explain brightness data.

\section{(2) The Boundary-Contour System and the}

\section{Feature-Contour System}

The theory asserts that two distinct types of edge, or contour, computations are carried out within two parallel systems. We call these systems the boundary-contour system and the feature-contour system. Boundary-contour signals are used to synthesize the boundaries, whether "real"'or "illusory," that the perceptual process generates. Feature-contour signals initiate the filling-in processes whereby brightnesses and colors spread until they either hit their first boundary contour or are attenuated due to their spatial spread. Boundary contours are not, in themselves, visible. They gain visibility by restricting the filling-in that is triggered by feature-contour signals.

These two systems obey different rules. The main rules can be summarized as follows.

\section{(3) Boundary Contours and Boundary \\ Completion}

The process whereby boundary contours are built up is initiated by the activation of oriented masks, or elongated receptive fields, at each position of perceptual space (Hubel \& Wiesel, 1977). Our perceptual analysis leads to the following hypotheses about how these masks activate their target cells, and about how these cells interact to generate boundary contours.

(a) Orientation and contrast. The output signals from the oriented masks are sensitive to the orientation and to the amount of contrast, but not to the direction of contrast, at an edge of a visual scene. Thus, a vertical boundary contour can be activated by either a close-to-vertical dark-light edge or a close-to-vertical light-dark edge at a fixed scenic position. The process whereby two likeoriented masks that are sensitive to direction of contrast at the same perceptual location give rise to an output signal that is not sensitive to direction of contrast is designated by a plus sign in Figure 1a.

(b) Short-range competition. (i) The cells that react to output signals due to like-oriented masks compete between nearby perceptual locations (Figure 1b). Thus, a mask of fixed orientation excites the like-oriented cells at its location and inhibits the like-oriented cells at nearby locations. In other words, an on-center off-surround organization of like-oriented cell interactions exists around each perceptual location. (ii) The outputs from this competitive stage input to the next competitive stage. At this stage, cells compete that represent perpendicular orientations at the same perceptual location (Figure 1c). This competition defines a push-pull opponent process. If a given orientation is inhibited, then its perpendicular orientation is disinhibited.

In all, a stage of competition between like orientations at different, but nearby, positions is followed by a stage

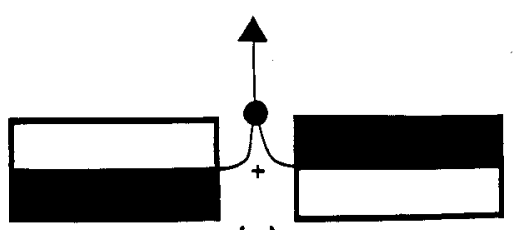

(a)

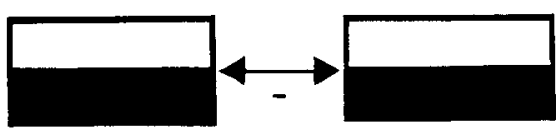

(b)
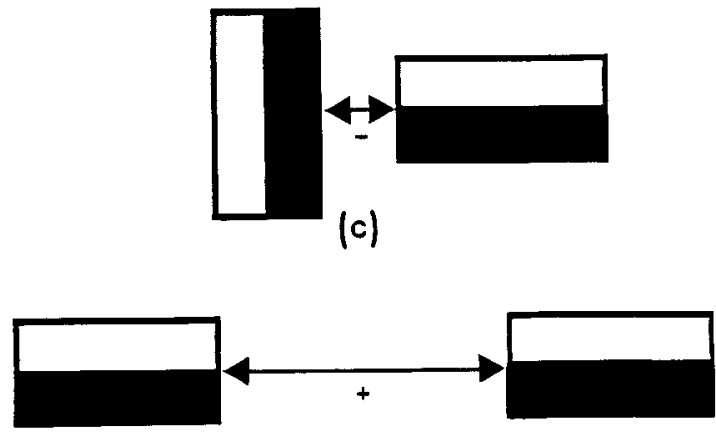

(d)

Figure 1. (a) Boundary-contour inputs are sensitive to the orientation and amount of contrast at a scenic edge, but not to its direction of contrast. (b) Like orientations compete at nearby perceptual locations. (c) Different orientations compete at each perceptual location. (d) Once activated, aligned orientations can cooperate across a larger visual domain to form real or illusory contours.

of competition between perpendicular orientations at the same position.

(c) Long-range oriented cooperation and boundary completion. The outputs from the last competitive stage input to a spatially long-range cooperative process that is called the "boundary-completion" process. Outputs due to like-oriented masks that are approximately aligned across perceptual space can cooperate via this process to synthesize an intervening boundary. The boundarycompletion process is capable of synthesizing global visual boundaries from local scenic contours (Grossberg \& Mingolla, 1985, in press). Both "real" and "illusory" boundaries are assumed to be generated by this boundarycompletion process.

Two simple demonstrations of a boundary-completion process with properties a-c can be made as follows. In Figure 2a, four Pac-man figures are arranged at the vertices of an imaginary rectangle. It is a familiar fact that an illusory Kanizsa (1974) square can be seen when all four Pac-man figures are black against a white background. The same is true when two Pac-man figures are black, the other two are white, and the background is gray, as in Figure $2 \mathrm{~b}$. The black Pac-man figures form darklight edges with respect to the gray background. The white Pac-man figures form light-dark edges with the gray background. The visibility of illusory edges around the illusory 

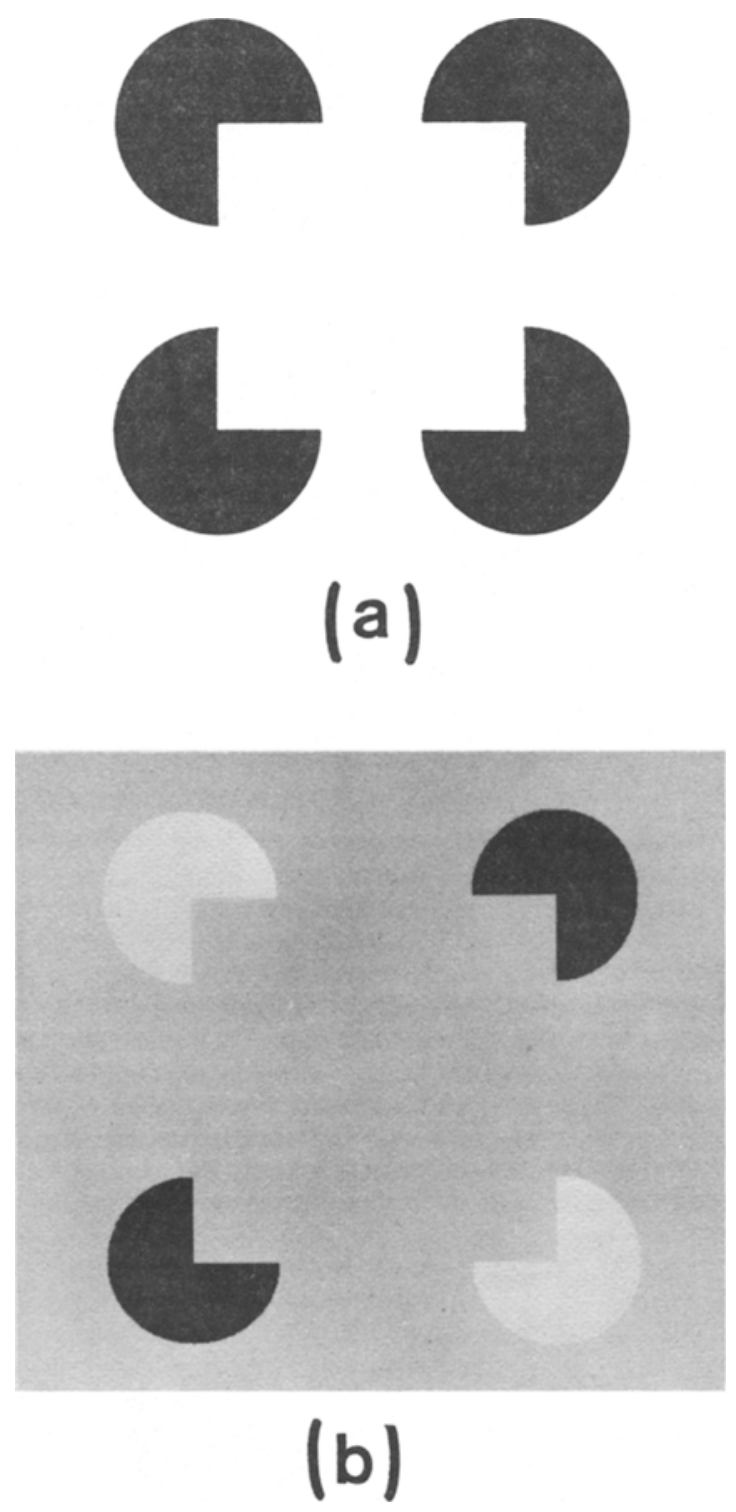

Figure 2. (a) An illusory Kanizsa square is induced by four black Pac-man figures. (b) An illusory square is induced by two black and two white Pac-man figures on a gray background. Illusory contours can thus join edges with opposite directions of contrast (The effect may be weakened by the photographic reproduction process.)

square shows that a process exists that is capable of completing contours between edges with opposite directions of contrast. This contour-completion process is thus sensitive to amount of contrast but not to direction of contrast.

Another simple demonstration of these contourcompleting properties can be constructed as follows. Divide a square into two equal rectangles along an imaginary boundary. Color one rectangle a uniform shade of gray. Color the other rectangle in shades of gray that progress from light to dark as one moves from end 1 of the rectangle to end 2 of the rectangle. Color end 1 a lighter shade than the uniform gray of the other rectan- gle, and color end 2 a darker shade than the uniform gray of the other rectangle. Then, as one moves from end 1 to end 2 , an intermediate gray region is passed whose luminance approximately equals that of the uniform rectangle. At end 1, a light-dark edge exists from the nonuniform rectangle to the uniform rectangle. At end 2, a dark-light edge exists from the nonuniform rectangle to the uniform rectangle. Despite this reversal in the direction of contrast from end 1 to end 2 , an observer can see an illusory edge that joins the two edges of opposite contrast and separates the intermediate rectangle region of equal luminance.

This boundary completion process, which seems so paradoxical when its effects are seen in Kanizsa squares, is also hypothesized to complete boundaries across the blind spot, across the faded images of stabilized retinal veins, and between all perceptual domains that are separated by sharp brightness or color differences.

(d) Binocular matching. A monocular boundary contour can be generated when a single eye views a scene. When two eyes view a scene, a binocular interaction can occur between outputs from oriented masks that respond to the same retinal positions of the two eyes. This interaction leads to binocular competition between perpendicular orientations at each position. This competition takes place at, or before, the competitive stage bii.

\section{(4) Feature Contours and Diffusive Filling-In}

The rules of contrast obeyed by the feature-contour process are different from those obeyed by the boundarycontour process.

(a) Contrast. The feature-contour process is insensitive to the orientation of contrast in a scenic edge, but it is sensitive to both the direction of contrast and the amount of contrast, unlike the boundary-contour process. For example, to compute the relative brightness across a scenic boundary, it is obviously important to keep track of which side of the scenic boundary has a larger reflectance. Sensitivity to direction of contrast is also needed to determine which side of a red-green scenic boundary is red and which is green. Due to its sensitivity to the amount of contrast, feature-contour signals "discount the illuminant."

In the simulations in this article, only one type of feature-contour signal is considered, namely, achromatic or light-dark signals. In the simulations of chromatic percepts, three parallel channels of double-opponent featurecontour signals are used: light-dark, red-green, and blueyellow. The simulations in this article consider only how input patterns are processed by a single network channel whose on-center off-surround spatial filter plays the role of a single spatial frequency channel (Grossberg, 1983b). We often call such a network a "spatial scale" for short. From our analysis of the dynamics of individual spatial scales, one can readily infer how multiple spatial scales, acting in parallel, transform the same input patterns.

The rules of spatial interaction that govern the feature- 
contour process are also different from those that govern the boundary-contour process.

(b) Diffusive filling-in. Boundary contours activate a boundary-completion process that synthesizes the boundaries that define monocular perceptual domains. Feature contours activate a diffusive filling-in process that spreads featural qualities, such as brightness or color, across these perceptual domains. Figure 3 depicts the main properties of this filling-in process.

It is assumed that featural filling-in occurs within a syncytium of cell compartments. By a syncytium of cells, we mean a regular array of cells in such an intimate relationship to one another that contiguous cells can easily pass signals between each other's compartment membranes. In the present instance, a feature-contour input signal to a cell of the syncytium activates that cell. Due to the syncytial coupling of this cell with its neighbors, the activity can rapidly spread to neighboring cells, then to neighbors of the neighbors, and so on. Since the spreading occurs via a diffusion of activity (Appendix A), it tends to average the activity that was triggered by the feature-contour input signal across the cells that receive this spreading activity. This averaging of activity spreads across the syncytium with a space constant that depends upon the electrical properties of both the cell interiors and their membranes. The electrical properties of the cell membranes can be altered by boundary-contour signals in the following way.

A boundary-contour signal is assumed to decrease the diffusion constant of its target cell membranes within the cell syncytium. It does so by acting as an inhibitory gating signal that causes an increase in cell membrane resistance (Appendix A). At the same time that a boundary-contour signal creates a barrier to the filling-in process at its target cells, it also acts to inhibit the activity of these cells. Thus, due to the physical process whereby a boundary contour limits featural spreading across the syncytium,

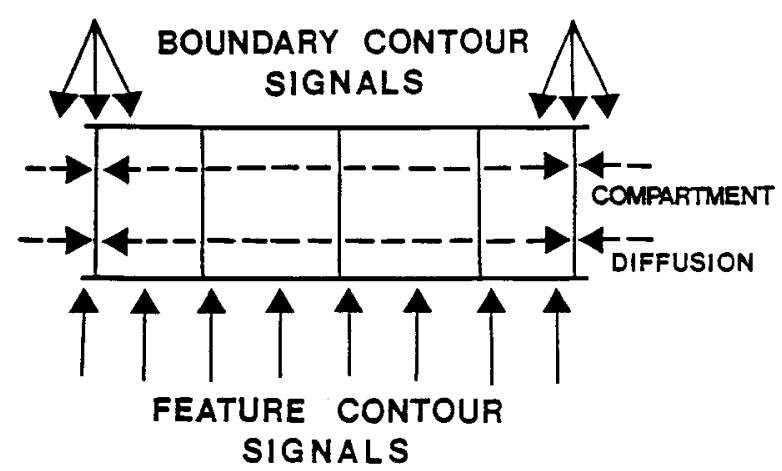

Figure 3. A monocular brightness and color stage (MBC): Monocular feature-contour signals activate cell compartments that permit rapid lateral diffusion of activity, or potential, across their boundaries, except at the boundaries that receive boundary-contour signals from the BCS stage of Figure 4. Consequently, the featurecontour signals are smoothed except at boundaries that are synthesized within the BCS stage. a boundary-contour input also acts as a feature-contour input to its target syncytial cells.

Such a diffusive filling-in reaction is hypothesized to instantiate featural filling-in over the blind spot, over the faded images of stabilized retinal veins, and over the illuminants that are discounted by feature-contour preprocessing.

Three distinguishable types of spatial interaction are implied by this description of the feature-contour system: (i) Spatial frequency preprocessing: Feature-contour signals arise as the outputs of several distinct on-center offsurround networks with different receptive field sizes, or spatial scales. (ii) Diffusive filling-in: The feature-contour signals within each spatial scale then cause activity to spread across scale's cell syncytium. This filling-in process has its own diffusive bandwidth. (iii) Figural boundaries: The boundary-contour signals define the limits of featural filling-in. Boundary contours are sensitive to the configuration of all edges in a scene, rather than to any single receptive field size. The interplay of these three types of spatial interaction will be essential in our explanations of brightness data.

\section{(5) Macrocircuit of Processing Stages}

Figure 4 describes a macrocircuit of processing stages into which the microstages of the boundary-contour system and feature-contour system can be embedded. The processes described by this macrocircuit are capable of synthesizing global properties of depth, brightness, and form information from monocularly and binocularly viewed patterns (Grossberg, 1983a, 1984). Table 1 lists the full names of the abbreviated macrocircuit stages, as well as the neural structures that seem most likely to execute analogous processes.

Each monocular preprocessing stage $\mathrm{MP}_{\mathrm{L}}$ and $\mathrm{MP}_{\mathrm{R}}$ can generate inputs to a boundary-contour system and a feature-contour system. The pathway $\mathrm{MP}_{\mathrm{L}} \rightarrow \mathrm{BCS}$ carries inputs to the left-monocular boundary-contour system. The pathway $\mathrm{MP}_{\mathbf{L}} \rightarrow \mathrm{MBC}_{\mathbf{L}}$ carries inputs to the leftmonocular feature-contour system. Only after all the microstages of scale-specific, orientation-specific, contrast-specific, competitive, and cooperative interactions (Section 3) take place within the BCS stage does this stage give rise to boundary-contour signals $B C S \rightarrow M B C_{L}$ that act as barriers to the diffusive filling-in triggered by $\mathrm{MP}_{\mathbf{L}} \rightarrow \mathrm{MBC}_{\mathbf{L}}$ feature-contour signals (Section 4). Thus, the divergence of the pathways $M_{L} \rightarrow M_{L} C_{L}$ and $M_{\mathrm{L}} \rightarrow \mathrm{BCS}$ allows the boundary-contour system and the feature-contour system to undergo significant processing according to different rules before their signals recombine within the cell syncytia.

\section{(6) FIRE: Resonant Lifting of Preperceptual} Data into a Form-in-Depth Percept

The activity patterns generated by feature-boundary interactions at the monocular brightness and color stages $M B C_{L}$ and $M B C_{R}$ must undergo further processing be- 


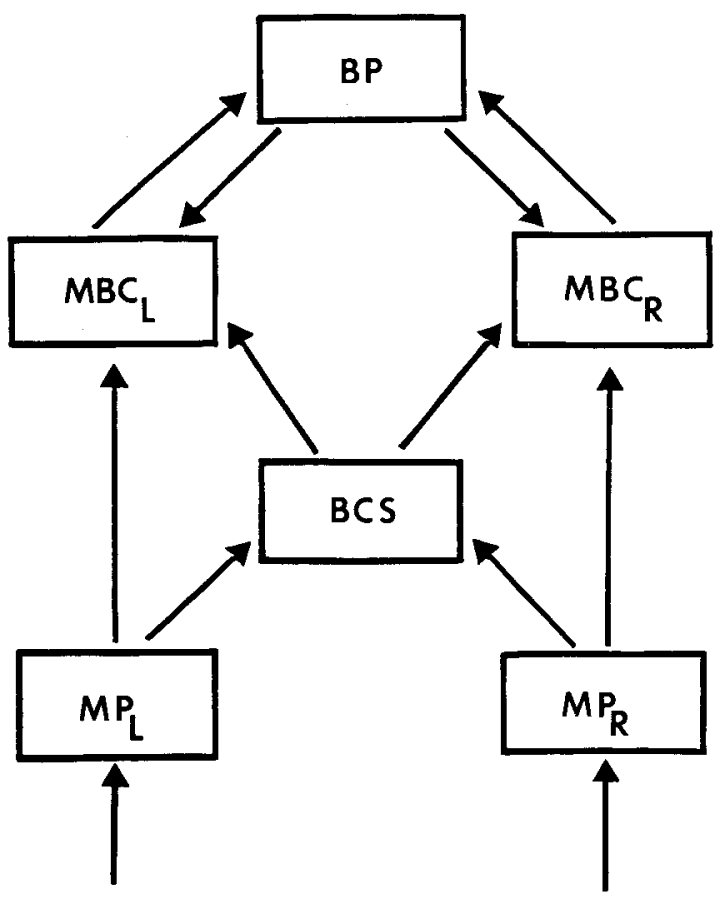

Figure 4. A macrocircuit of processing stages: Table 1 lists the functional names of the abbreviated stages and indicates a neural interpretation of these stages. Boundary-contour formation is assumed to occur within the BCS stage. Its output signals to the monocular $\mathrm{MBC}_{\mathrm{L}}$ and $\mathrm{MBC}_{\mathrm{R}}$ stages define boundaries within which feature-contour signals from $M P_{L}$ or $M P_{R}$ can trigger the spreading, or diffusion, of featural quality.

fore they can be perceived. This property is analogous to the fact that a contoured monocular image is not always perceived. It can, for example, be suppressed by a discordant image to the other eye during binocular rivalry. Only activity patterns at the binocular percept (BP)

Table 1

Names of Macrocircuit Stages

\begin{tabular}{|c|c|}
\hline Abbreviations & Full Names \\
\hline $\mathrm{MP}_{\mathrm{L}}$ & $\begin{array}{l}\text { Left Monocular Preprocessing Stage } \\
\text { (Lateral geniculate nucleus) }\end{array}$ \\
\hline $\mathrm{MP}_{\mathrm{R}}$ & $\begin{array}{l}\text { Right Monocular Preprocessing Stage } \\
\text { (Lateral geniculate nucleus) }\end{array}$ \\
\hline BCS & $\begin{array}{l}\text { Boundary Contour Synthesis Stage } \\
\text { [Interactions initiated by the hypercolumns in striate } \\
\text { cortex-Area } 17 \text { (Hubel and Wiesel, 1977)] }\end{array}$ \\
\hline $\mathrm{MBC}_{\mathrm{L}}$ & $\begin{array}{l}\text { Left Monocular Brightness and Color Stage } \\
\text { [Interactions initiated by the cytochrome oxydase stain- } \\
\text { ing blobs--Area } 17 \text { (Hendrickson, Hung, \& Wu, 1981; } \\
\text { Horton \& Hubel, 1981; Hubel \& Livingstone, 1981; } \\
\text { Livingstone \& Hubel, 1982)] }\end{array}$ \\
\hline $\mathrm{MBC}_{\mathbf{R}}$ & $\begin{array}{l}\text { Right Monocular Brightness and Color Stage } \\
\text { (Interactions initiated by the cytochrome oxydase stain- } \\
\text { ing blobs-Area 17) }\end{array}$ \\
\hline $\mathrm{BP}$ & $\begin{array}{l}\text { Binocular Percept Stage } \\
\text { [Area V4 of the prestriate cortex (Zeki, 1983a, 1983b)] }\end{array}$ \\
\hline
\end{tabular}

stage of Figure 4 are perceived. Signals from stage $M B C_{L}$ and/or stage $\mathrm{MBC}_{\mathrm{R}}$ that are capable of activating the $\mathrm{BP}$ stage are said to "lift" the preprocessed monocular patterns into the perceptual domain (Cohen \& Grossberg, 1984; Grossberg, 1983b). We use the word "lift" instead of a word like "search" because the process occurs directly via a single parallel processing step, rather than by some type of serial algorithm. This lifting process works as follows.

Monocular arrays of cells in $\mathrm{MBC}_{\mathrm{L}}$ and $M \mathrm{MBC}_{\mathrm{R}}$ send topographically organized pathways to $\mathrm{BP}$ and receive topographically organized pathways from BP. A monocular activity pattern across $\mathrm{MBC}_{\mathrm{L}}$ can elicit output signals in the $\mathrm{MBC}_{\mathrm{L}} \rightarrow \mathrm{BP}$ pathway only from positions that are near contours, or edges, of the $\mathrm{MBC}_{\mathrm{L}}$ activity pattern (Figure 5). Contours of a $\mathrm{MBC}_{\mathrm{L}}$ pattern must not be confused with edges of an external scene. They are due to boundary-contour signals in the $\mathrm{BCS} \rightarrow \mathrm{MBC}_{\mathrm{L}}$ pathway, which themselves are the result of a great deal of preprocessing. Thus, no contour signals are initially elicited from the $\mathrm{MBC}_{\mathrm{L}}$ stage to the BP stage at positions within the interiors of filled-in regions. Similar remarks hold for contour signals from the $\mathrm{MBC}_{\mathrm{R}}$ stage to the $\mathrm{BP}$ stage.

Pairs of contour signals from $\mathrm{MBC}_{\mathrm{L}}$ and $\mathrm{MBC}_{\mathrm{R}}$ that correspond to similar perceptual locations are binocularly matched at the BP stage. If both contour signals overlap sufficiently, then they can form a fused binocular contour with the BP stage. If their positions mismatch by a larger amount, then both contours can mutually inhibit each other, or the stronger contour can suppress the weaker contour. If their positions are even more disparate, then a pair, or "double image," of contours can be activated at the BP stage. These possibilities are due to the fact that the contour signals from $M B C_{L}$ and $M B C_{R}$ to BP possess an excitatory peak surrounded by a pair of inhibitory troughs. Under conditions of monocular viewing, the contour signals from (say) $\mathrm{MBC}_{L}$ to $\mathrm{BP}$ are always registered, or "self-matched," at BP because no contours exist from $\mathrm{MBC}_{\mathrm{R}}$ that are capable of suppressing them.

Contours at the BP stage that survive this binocular matching process can send topographic contour signals back to $M B C_{L}$ and $M B C_{R}$ along the feedback pathways (Figure 5). Remarkably, feedback exchange of such local contour signals can trigger a rapid filling-in reaction across thousands of cells. This filling-in reaction is due to the form of the contour signals that are fed back from $B P$ to $M B C_{L}$ and $M B C_{R}$. These signals also possess an excitatory peak surrounded by a pair of inhibitory troughs. The inhibitory troughs cause local nonuniformities in the activity pattern near the original $\mathrm{MBC}_{\mathrm{L}}$ or $\mathrm{MBC}_{\mathrm{R}}$ contour. These local nonuniformities are seen by the $\mathrm{MBC}_{\mathrm{L}} \rightarrow \mathrm{BP}$ and $\mathrm{MBC}_{\mathrm{R}} \rightarrow \mathrm{BP}$ pathways as new contiguous contours, which can thus send signals to BP. In this way, a matched contour at BP can trigger a standing wave of activity that can rapidly spread, or fill in, across BP 


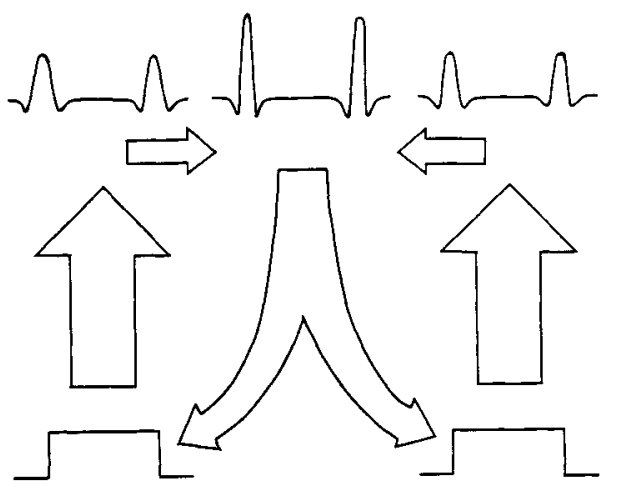

Figure 5. Binocular representation of $\mathrm{MBC}$ patterns at the $\mathrm{BP}$ stage: Each $\mathrm{MBC}_{L}$ and $\mathrm{MBC}_{\mathrm{R}}$ activity pattern is filtered in such a way that its contours generate topographically organized inputs to the BP stage. At the BP stage, these contour signals undergo a process of binocular matching. This matching process takes place simultaneously across several on-center off-sourround networks, each with a different spatial interaction bandwidth. Contours capable of matching at the BP stage send feedhack signals to their respective $\mathrm{MBC}_{\mathrm{L}}$ or $\mathrm{MBC}_{\mathrm{R}}$ patterns. Closing this feedback loop of local edge signals initiates the rapid spreading of a standing wave that resonantly "lifts" a binocular representation of the matched monocular patterns into the BP stage. This standing wave, or filling-in resonant exchange (FIRE), spreads until it hits the first binocular mismatch within its spatial scale. The ensemble of all resonant standing waves across the multiple spatial scales of the BP stage constitutes the network percept. If all $\mathrm{MBC}_{\mathrm{L}}$ or $\mathrm{MBC}_{\mathrm{R}}$ contour inputs are suppressed by binocular matching at a spatial scale of the BP stage, then their respective monocular activity patterns cannot be lifted into resonant activity within this BP spatial scale. The BP spatial scales selectively resonate with some, but not all, monocular patterns within the $\mathrm{MBC}_{\mathrm{L}}$ and $\mathrm{MBC}_{\mathrm{R}}$ stages.

until it hits the first pair of mismatched contours. Such a mismatch creates a barrier to filling-in. As a result of this filling-in process across BP, the activities at interior positions of filled-in regions of $M B C_{L}$ and $M B C_{R}$ can be lifted into perception within BP. Although such an interior cell in $\mathrm{MBC}_{\mathrm{L}}$ sends topographic signals to $\mathrm{BP}$, these signals are not topographically related to $M P_{L}$ in a simple way, due to syncytial filling-in within $\mathrm{MBC}_{\mathrm{L}}$.

The properties of the resonant filling-in reaction imply that $M B C_{L}$ or $M_{B} C_{R}$ activity patterns that do not emit any contour signals to BP cannot enter perception. Activity patterns, all of whose contour signals are inhibited within BP due to binocular mismatch, also cannot enter perception. Only activity patterns that lie between a contour match and its nearest contour mismatch can enter perception.

Such a filling-in reaction, unlike diffusive filling-in (Section 4), is a type of nonlinear resonance phenomenon, which we call a "filling-in resonant exchange" (FIRE). In the full theory, multiple networks within $M B C_{L}$ and $M B C_{R}$ that are sensitive to different spatial frequencies and disparities are topographically matched within multiple networks of BP. The ensemble of all such resonant standing waves constitutes the network's percept. Cohen and Grossberg (1984) and Grossberg (1983b) describe how these ensembles encode global aspects of depth, brightness, and form information. In this article, we show that these ensembles also mimic data about Fechner's paradox, binocular brightness summation, and binocular brightness averaging (Sections 13-15). The fact that a single process exhibits all of these properties enhances the plausibility of the rules whereby FIRE contours are computed and matched within BP. The standing waves in the BP stage may themselves be further transformed, say by a local smoothing operation. This type of refinement does not alter our discussion of binocular brightness data; hence, it will not be further discussed.

\section{(7) Binocular Rivalry, Stabilized Images, and the Ganzfeld}

The following qualitative properties of the FIRE process illustrate how binocular rivalry and the fading of ganzfelds and stabilized images can occur within the network of Figure 4.

Suppose that, due to binocular matching of perpendicular orientations, as in Section 3d, some left-monocular boundary contours are suppressed within the BCS stage. Then these boundary contours cannot send boundarycontour signals to the corresponding region of stage $M C_{L}$. Featural activity thus quickly diffuses across the network positions corresponding to these suppressed contours (Gerrits \& Vendrick, 1970). Consequently, no contour output signals can be emitted from these positions within the $\mathrm{MBC}_{\mathrm{L}}$ stage to the BP stage. No edge matches within the BP stage can occur at these positions, so no effective feedback signals are returned to the $M B C_{L}$ stage at these positions to lift the corresponding monocular subdomain into perception. Thus, the subdomains whose boundary contours are suppressed within the BCS stage are not perceived. As soon as these boundary contours win the BCS binocular competition, their subdomain contours can again rapidly support the resonant lifting of the subdomain activity pattern into perception at the BP stage. During binocular rivalry, an interaction between rapidly competing short-term memory traces and slowly habituating transmitter gates can cause oscillatory switching between left and right BCS contours (Grossberg, 1980, 1983a).

The same argument shows that a subdomain is not perceived if its boundary edges are suppressed by binocular rivalry within the BCS stage or by image stabilization, or if they simply do not exist, as in a ganzfeld.

\section{(8) The Interplay of Controlled}

\section{and Automatic Processes}

The most significant technical insights that our theory introduces concern the manner in which local computations can rapidly generate global context-sensitive representations via hierarchically organized networks whose individual stages undergo parallel processing. Using these insights, one can also begin to understand how internally generated "cognitive" feature-contour signals or "cognitive" boundary-contour signals can modify the global representations generated within the network of 
Figure 4 (Gregory, 1966; Grossberg, 1980). Indeed, the network does not know which of its contour signals are generated internally and which are generated externally. One can also now begin to understand how state-dependent nonspecific changes in sensitivity at the various network stages (e.g., attentional shifts) can modify the network's global representations. For example, the contrast sensitivity of feature-contour signals can change as a function of background input intensity or internal nonspecific arousal (Grossberg, 1983b, Sections 24-28). The balance between direct feature-contour signals and diffusive filling-in signals can thus be altered by changes in input luminance or arousal parameters, and can thereby influence how well filling-in can overcome feature-contour contrast effects during the Craik-O'Brien illusion (Section 9).

Once such internally or externally controlled factors are specified, however, the network automatically generates its global representations using the intrinsic structure of its circuitry. In all aspects of our theoretical work, controlled and automatic factors participate in an integrated network design (Grossberg, 1982a), rather than forming two computationally disjoint serial and parallel subsystems, as Schneider and Shiffrin (1977) have suggested. Even the complementary attentional and orienting subsystems that have been hypothesized to regulate the stability and plasticity of long-term memory encoding processes in response to expected and unexpected events (Grossberg, 1975, 1982a, 1982b) both utilize parallel mechanisms that are not well captured by the controlled vs. automatic processing dichotomy.

\section{(9) Craik-O'Brien Luminance Profiles and}

\section{Multiple Step Illusions}

Arend et al. (1971) have studied the perceived brightness of a variety of luminance profiles. The construction of these profiles was suggested by the seminal article of O'Brien (1958). Each of the luminance profiles was produced by placing appropriately cut sectors of black and white paper on a disk. The disk was rotated at a rate much faster than that required for flicker fusion. The luminances thereby generated were then independently calibrated. The subjects were asked to describe the relative brightness distribution by describing the locations and directions of all brightness changes, and by ordering the brightnesses of regions that appeared uniform. Ordinal, rather than absolute, brightness differences were thereby determined.

One of their important results is schematized in Figure 6. Figure 6a describes a luminance profile in which two Craik-O'Brien luminance cusps are joined to $\mathrm{a}$ uniform background luminance. The luminances to the left and to the right of the cusps are equal, and the average luminance across the cusps equals the background luminance. Figure $6 \mathrm{~b}$ shows that this luminance profile is perceived as (approximate) steps of increasing brightness. In particular, the perceived brightnesses of the left and right backgrounds are significantly different, despite the fact that their luminances are equal.

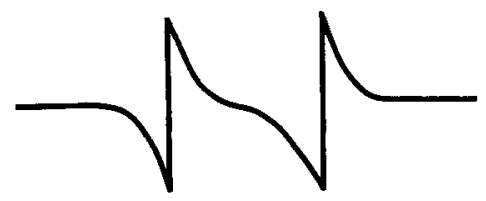

(a)

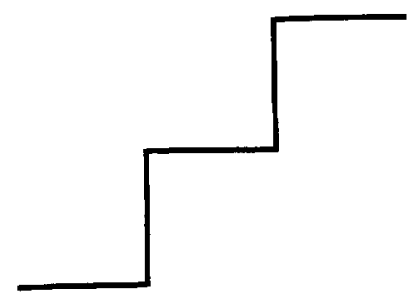

(b)

Figure 6. (a) A one-dimensional slice across a two-dimensional Craik-O'Brien luminance profile. The background luminances at the left and right sides of the profile are equal. (b) This luminance profile appears like a series of two (approximate) steps in increasing brightness.

This type of result led Arend et al. (1971, p. 369) to conclude that "the brightness information generated by moving contours is difference information only, and the absolute information hypothesis is rejected." In other words, the nonuniform luminances between successive edges are discounted, and only the luminance differences of the edges determine the percept. Similar concepts were developed by Land (1977).

This conclusion does not explain how the luminance differences at the edges are computed, or how the edges determine the subjective appearance of the perceptual domains that exist between the edges. The incomplete nature of the conclusions does not, however, limit their usefulness as a working hypothesis. This hypothesis must, however, be tempered by the fact that it is not universally true. For example, the hypothesis does not explain illusory brightness differences that can exist along illusory contours that cross regions of uniform luminance (Kanizsa, 1974; Kaufman, 1974; Kennedy, 1979). It does not explain how Craik-O'Brien filling-in can improve or deteriorate as the balance between background illumination and edge contrast is varied (Heggelund \& Krekling, 1976; van den Brink \& Keemink, 1976). It does not explain why a strong Craik-O'Brien effect is seen when a vertical computer-generated luminance cusp on a uniform background is enclosed by a black border that touches the two ends of the cusp, yet vanishes completely when the black border is removed and the cusp is viewed within a uniform background on all sides (Todorović, 1983). It does not explain why, in response to five cusps rather than two, subjects may see a flattened percept rather than five rising steps (Coren, 1983). The present theory suggests 
an explanation of all these properties. The illusory brightness properties are discussed in Grossberg (1984) and Grossberg and Mingolla (in press). The remaining issues are clarified below.

Figure 7 describes the results of a computer simulation of the two-step brightness illusion that is described in Figure 6. The networks of differential equations on which the simulation is based are summarized in Appendix A. Figure 7 depicts equilibrium solutions to which these networks of differential equations rapidly converge. All of the simulation results reported herein are equilibrium so- lutions of such networks. These networks define onedimensional arrays of cells due to the one-dimensional symmetry in the luminance profiles.

Figure 7a describes the input pattern to the network. The double cusps are surrounded by a uniform luminance level that is Guassianly smoothed at its edges to minimize spurious edge effects. Figure $7 b$ shows that each of the two luminance cusps in the input pattern generates a narrow boundary-contour signal. Each boundary-contour signal causes a reduction in the rate of diffusion across the membranes of its target cells at the $\mathrm{MBC}_{\mathrm{L}}$ or $\mathrm{MBC}_{\mathrm{R}}$ stage.

\section{TWO STEP ILLUSION}

INPUT PATTERN

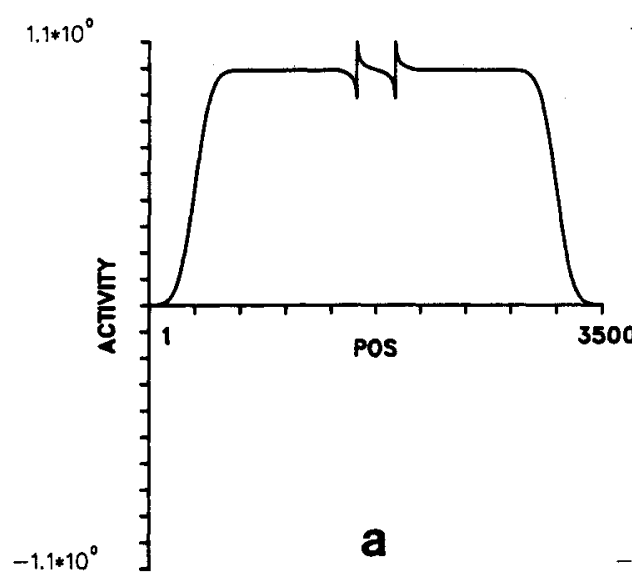

FEATURE CONTOUR PATTERN

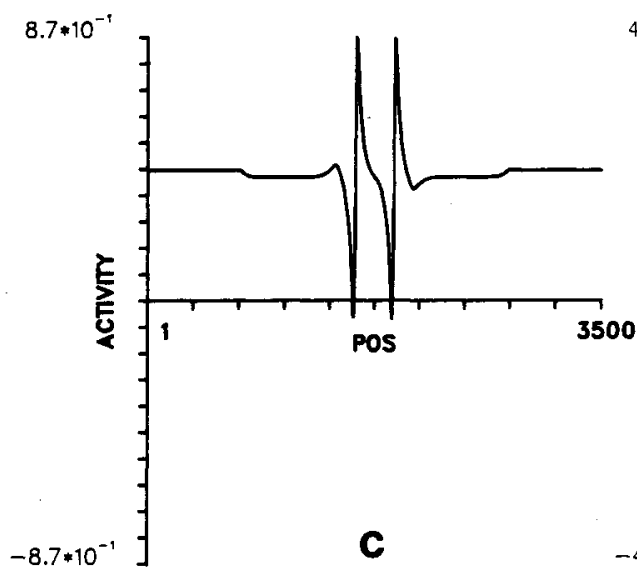

BOUNDARY CONTOUR PATTERN
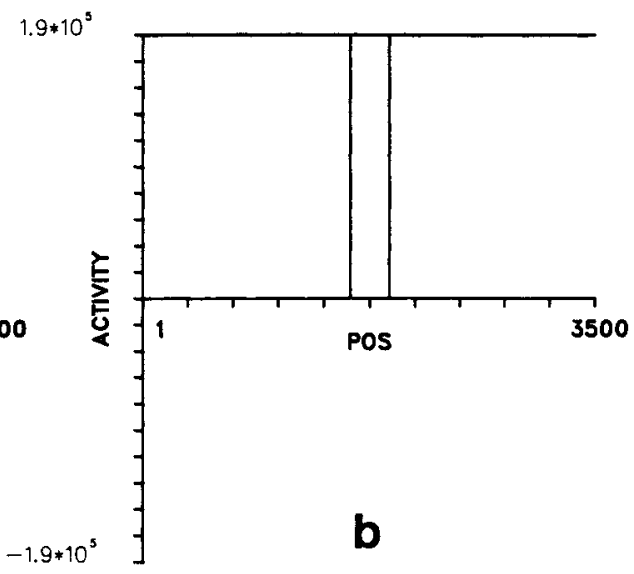

MONOCULAR BRIGHTNESS PATTERN

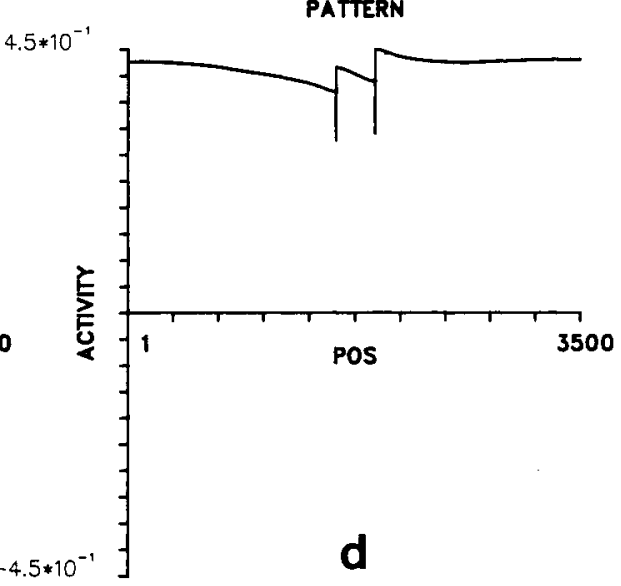

Figure 7. Simulation of the two-step illusion: (a) Input luminance pattern. (b) The pattern of diffusion coefficients that is induced by boundary contours. This pattern determines the limits of featural spreading across the cell syncytium. The two luminance cusps in (a) determine a pair of boundary contours at which the diffusion coefficients are small in (b). (c) The feature-contour pattern induced by (a). The background luminance is attenuated, and the relative contrasts of the luminance cusps are accentuated. (d) When pattern c diffuses within the syncytial domains determined by (b), a series of two approximate steps of activity results. 
A reduced rate of diffusion prevents the lateral spread of featural activity across the membranes of the affected cells. A reduced diffusion rate thereby dynamically generates boundary contours within the cell syncytium (Figure 3). Successive boundary contours determine the spatial domains within which featural activity can spread.

The feature-contour process attenuates the background luminance of the input pattern and computes the relative contrasts of the cusps. It does this by letting the individual inputs interact within a shunting on-center off-surround network (Grossberg, 1983b). Such a network is defined in Appendix A, Equation 1. The resultant feature-contour activity pattern is an input pattern to a cell syncytium. The boundary-contour signals from the BCS stage also contribute to this input pattern. Boundary-contour signals generate feature-contour signals as well as boundarycontour signals because they increase cell membrane resistances in order to decrease the cells' diffusion constants, as described in Section 4b. Due to this effect on cell-membrane resistances, boundary-contour signals are a source of inhibitory feature-contour signals. These inhibitory signals act on a narrower spatial scale than the feature-contour signals from the $M P_{L}$ and $M P_{R}$ stages. The total feature-contour input pattern received by $M B C_{L}$ is the sum of the feature-contour patterns from the $\mathbf{M P}_{\mathbf{L}}$ and BCS stages. This total feature-contour input pattern is depicted in Figure 7c. (The flanks of this pattern were artificially extended to the left and to the right to avoid

\section{FIVE STEP ILLUSION}
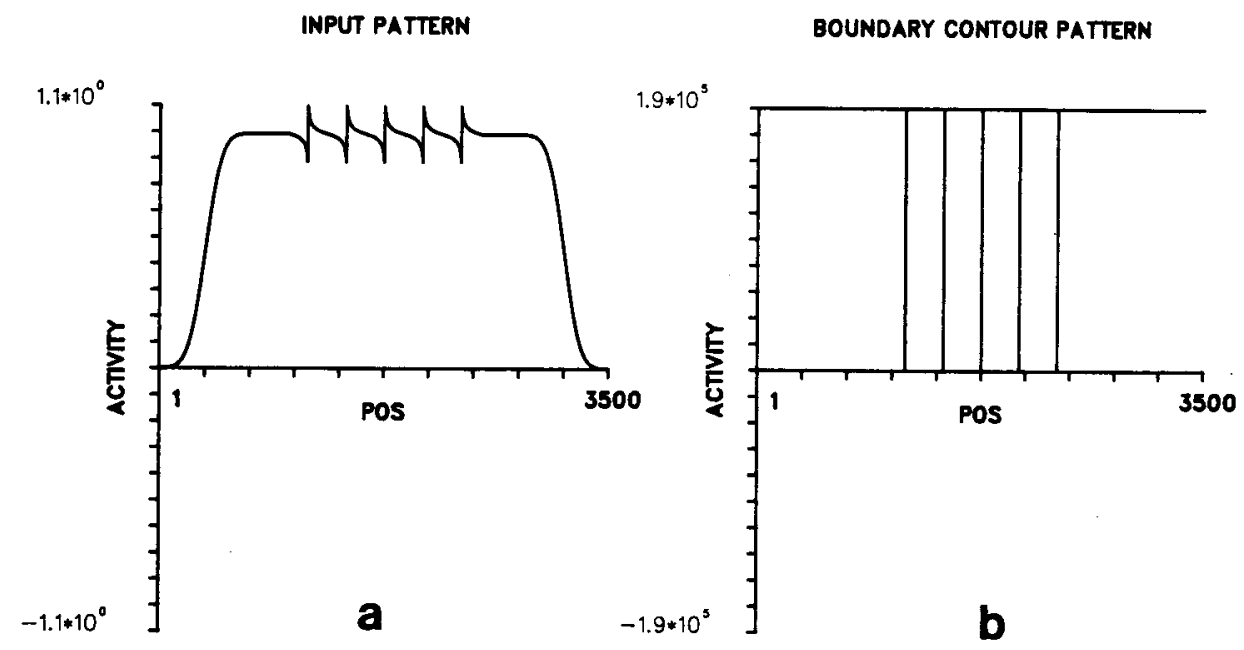

FEATURE CONTOUR PATTERN

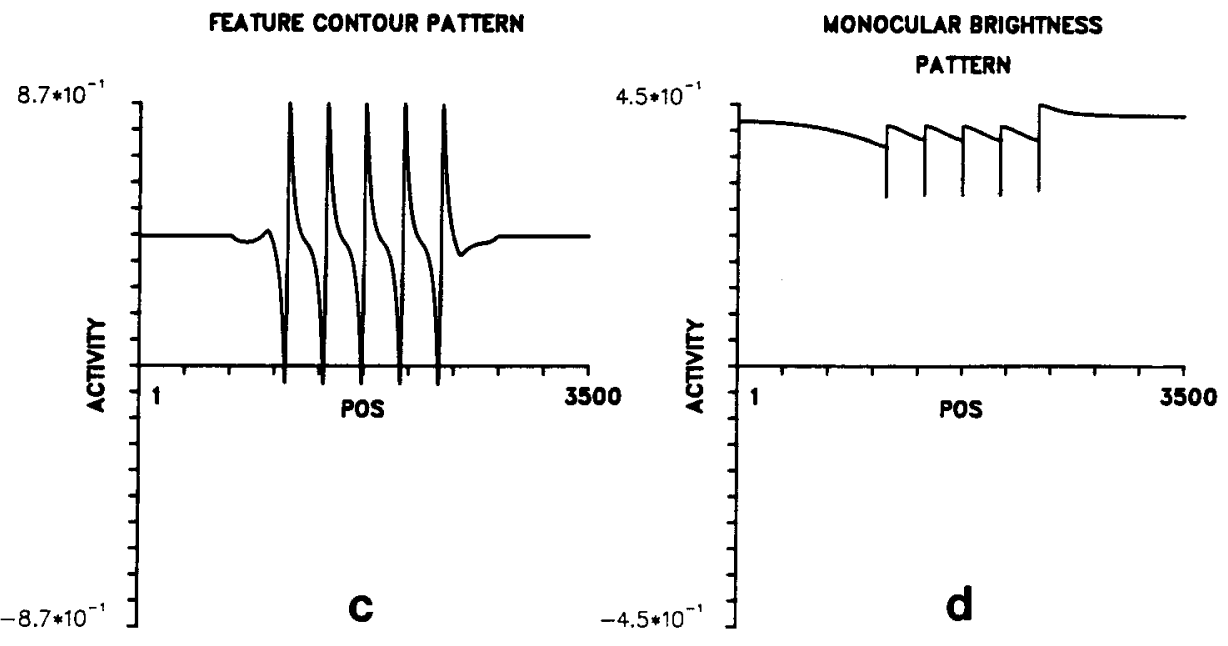

Figure 8. Simulation of the five-step illusion: The main difference between Figures $7 \mathrm{~b}$ and $8 \mathrm{~b}$ is that Figure $8 \mathrm{~b}$ contains six syncytial domains whereas Figure $7 \mathrm{~b}$ contains only three. Each domain averages only the part of the feature-contour pattern that it receives. The result in Figure $8 \mathrm{~d}$ is a much flatter pattern than one might expect from Figure 7d. 
spurious boundary effects and to simulate the output when the input pattern is placed on an indefinitely large field.) When the feature-contour input pattern of Figure $7 \mathrm{c}$ is allowed to diffuse within the perceptual domains defined by the boundary-contour pattern of Figure $7 \mathrm{~b}$, the steplike activity pattern of Figure $7 \mathrm{~d}$ is the result.

Figure 8 simulates a luminance profile with five cusps, using the same equations and parameters that generate Figure 7 . The activity pattern in Figure 8d is much flatter than one might expect from the step-like pattern in Figure $7 \mathrm{~d}$. Coren (1983) found a similar result with this type of stimulus. Figure 7 suggests that the result of Coren (1983), which he attributes to cognitive factors, may be partially explained by feature-contour and boundarycontour interactions due to a single spatial scale.

Such a single-scale reaction does not, however, exhaust even the noncognitive monocular interactions that are hypothesized to occur within our theory. The existence of multiple spatial scales has been justified from several points of view (Graham, 1981; Graham \& Nachmias, 1971; Grossberg, 1983b; Kaufman, 1974; Kulikowski, 1978). The influence of these multiple scale reactions are also suggested by some displays of Arend et al. (1971). One such display is redrawn in Figure 9. The transformation of cusp in Figure 9a into step in Figure $9 \mathrm{~b}$ and the computation of the relative contrast of the increments on their backgrounds are easy for the single-scale network that simulates Figures 7 and 8. This network cannot, however, generate the same brightness on both sides of the increments in Figure 9b, because the boundarycontour signals due to the increments prevent the featurecontour signals due to the cusps from diffusing across the increments. Thus, to a single-scale network, the left and right distal brightnesses appear more equal than the brightnesses on both sides of the cusps.

\section{(a)}

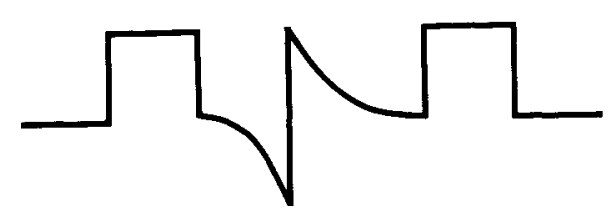

(b)

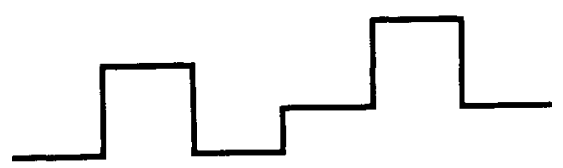

Figure 9. The luminance profile in (a) generates the brightness profile in (b). (Redrawn with permission from Arend, Buehler, \& Lockhead, 1971.)
This difficulty is partially overcome when multiple spatial scales (viz, separate shunting on-center off-surround networks with different intercellular interaction coefficients) process the same input pattern, and the perceived brightness is derived from the average of all the resultant activity patterns across their respective syncytia. In this setting, a low-frequency spatial scale may generate a boundary contour in response to the cusp, but not in response to the increments (Grossberg, 1983b). The monocular brightness pattern generated by such a scale is thus a single step centered at the position of the cusp. When this step is averaged with the monocular brightness pattern of a high-spatial-frequency scale, the difference between proximal and distal background brightness estimates becomes small relative to the difference between step and background brightnesses. This explanation of Figure 9 may be testable by selectively adapting out the high- or low-spatial-frequency scales.

The action of low-spatial-frequency scales can also contribute to the flattening of the perceived brightnesses induced by a five-cusp display. Five cusps activate a broader network domain than do two cusps of equal size. Lowspatial-frequency scales that do not significantly react to two cusps may generate a blob-like reaction to five cusps. When such a reaction is averaged in with the already flattened high-spatial-frequency reaction, an even flatter percept can result.

\section{(10) Smoothly Varying Luminance Contours vs. Steps of Luminance Change}

Bergström (1966, 1967a, 1967b) has collected data that restrict the generality of the conclusion that sharp edges control the perception of brightness. In those experiments, he compared the relative brightness of several luminance displays. Some of the displays possessed no sharp luminance edges within their interiors. Other displays did possess sharp luminance edges. Bergström used a variant of the rotating prism method to construct twodimensional luminance distributions in which the luminance changed in the horizontal direction but was constant in each narrow vertical strip. The horizontal changes in two such luminance distributions are shown in Figure 10.

Figure 10a depicts a luminance profile wherein the luminance continuously decreases from left to right. Bergström constructed this profile to quantitatively test the theory of Mach (1866) that attributes brightness changes to the second derivative $\mathrm{d}^{2} \mathrm{~L}(\mathrm{x}) / \mathrm{dx}^{2}$ with respect to the spatial variable $x$ of the luminance profile $L(x)$ (see Ratliff, 1965). Mach (1866) concluded that, if two adjacent points $\mathrm{x}_{1}$ and $\mathrm{x}_{3}$ have similar luminances $\left[\mathrm{L}\left(\mathrm{x}_{1}\right) \approx \mathrm{L}\left(\mathrm{x}_{3}\right)\right]$, then the point $x_{3}$ at which the second derivative is negative $\left\{\left[\mathrm{d}^{2}\left(\mathrm{x}_{3}\right) / \mathrm{dx}^{2}\right]<0\right\}$, looks brighter than the point $\mathrm{x}_{1}$ at which the second derivative is positive $\left\{\left[\mathrm{d}^{2} \mathrm{~L}\left(\mathrm{x}_{1}\right) / \mathrm{dx}^{2}\right]>\right.$ $0\}$, and that a transition between a darker and a lighter percept occurs at the intervening inflection point $\mathrm{x}_{2}$ $\left\{\left[\mathrm{d}^{2} \mathrm{~L}\left(\mathrm{x}_{2}\right) / \mathrm{dx}^{2}\right]=0\right\}$. In Figure 11a, as Mach would predict, the position $x_{3}$ to the right of $x_{2}$ looks brighter than the position $x_{1}$ to the left of $x_{2}$. Figure 11a describes 

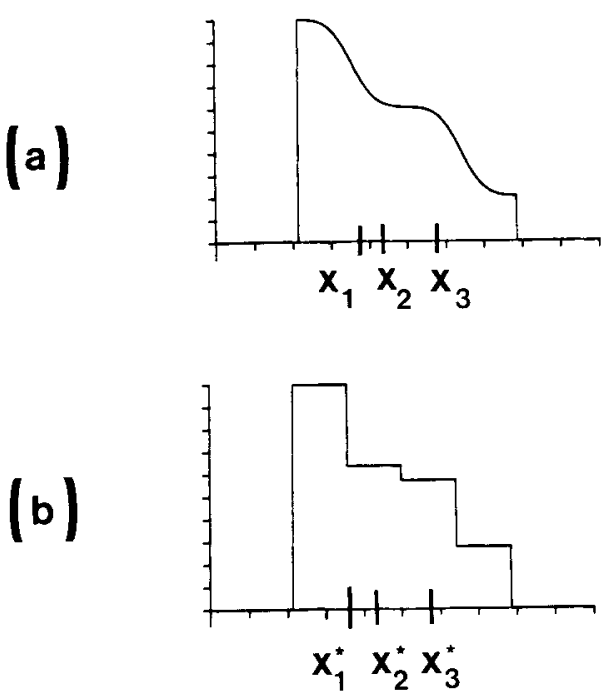

Figure 10. Two luminance profiles studied by Bergström. Position $x_{3}$ of (a) looks brighter than position $x_{3}^{*}$ of (b). Also position $x_{3}$ looks brighter than position $x_{1}$ in (a), and position $x_{3}^{*}$ looks somewhat brighter than position $x_{1}^{*}$ in (b). These data challenge the hypothesis that sharp edges determine the level of brightness. They also challenge the hypothesis that a sum of spatial-frequency-filtered patterns determines the level of brightness.

the results of a magnitude-estimation procedure that was used to determine the brightnesses of different positions along the luminance profile. For details of this procedure, Bergström's original articles should be consulted.

Figure 11a challenges the hypothesis that brightness perception depends exclusively upon difference estimates at sharp luminance edges. No edge exists at the inflection point $\mathrm{x}_{2}$, yet a significant brightness difference is generated around position $x_{2}$. Moreover the brightness difference inverts the luminance gradient, since $x_{1}$ is more luminous than $x_{3}$, yet $x_{3}$ looks brighter than $x_{1}$.

One might attempt to escape this problem by claiming that, although the luminance profile in Figure 10a contains no manifest edges, the luminance changes sufficiently rapidly across space to be edge-like with respect to some spatial scale. This hypothesis collapses when the luminance profile of Figure $10 \mathrm{~b}$ is considered. The luminance profile of Figure $10 \mathrm{~b}$ is constructed from the luminance profile of Figure 10a as follows. The luminance in each rectangle of Figure $10 \mathrm{~b}$ is the average luminance taken across the corresponding positions of Figure 10a. Unlike Figure 10a, however, Figure $10 \mathrm{~b}$ possesses several sharp edges. If the hypothesis of Arend et al. (1971) is taken at face value, then position $x_{3}^{*}$ of Figure 10b should look brighter than position $x_{3}$ of Figure 10a. This is because mean luminances are preserved between the two figures and Figure $10 \mathrm{~b}$ has sharp edges, whereas Figure 10a has no interior edges whatsoever.

A magnitude estimation procedure yielded the data shown in Figure 11b. Comparison of Figures 11a and 11b shows that position $\mathrm{x}_{3}^{*}$ looks darker, not brighter, than position $\mathrm{x}_{\mathbf{3}}$. These data cast doubt on the conclusion of Arend et al. (1971), just as the data of Arend et al. cast doubt on the conclusion of Mach (1866).

Our numerical simulations reproduce the main effects summarized in Figures 10 and 11. The critical feature of these simulations is that the two luminance profiles in Figure 10 generate different boundary-contour patterns as well as different feature-contour patterns. The luminance profile of Figure 12a generates boundary contours only at the exterior edges of the luminance profile (Figure 12b). By contrast, each interior step of luminance of Figure 13a also generates a boundary contour (Figure 13b). Thus, the monocular perceptual domains that are defined by the two luminance profiles are entirely different. In this sense, the two profiles induce, and are processed by, different perceptual spaces. These different parsings of the cell syncytium not only define different numbers of spatial domains, but also different sizes of domains over which featural quality can spread.

In addition, the smooth vs. sharp contours in the two luminance profiles generate different feature-contour patterns (Figures 12c and 13c). The differences between the feature-contour patterns do not, however, explain Bergström's data, because the feature-contour pattern at position $\mathrm{X}_{3}^{*}$ in Figure $13 \mathrm{c}$ is more intense than the featurecontour pattern at position $x_{3}$ in Figure 12c. This is the result one would expect from classical analyses of contrast enhancement. By contrast, when these featurecontour patterns are diffusively averaged between their respective boundary contours, the result of Bergström is obtained. The monocular brightness pattern at position $\mathrm{x}_{3}$ in Figure 12d is more intense than the monocular brightness pattern at position $x_{3}^{*}$ in Figure 13d. We therefore concur with Bergström in his claim that these results are paradoxical from the viewpoint of classical notions of brightness contrast. We know of no other brightness

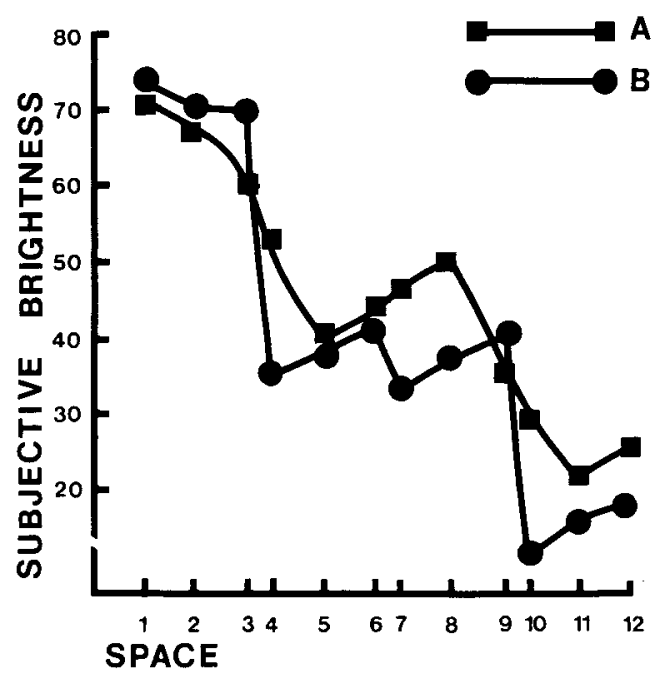

Figure 11. Magnitude estimates of brightness in response to the luminance profiles of Figure 10. (Redrawn from Bergström, 1966.) 
theory that can provide a principled explanation of both the Arend et al. (1971) data and the Bergström (1966, 1967a, 1967b) data.

In particular, both types of data cause difficulties for the Fourier theory of visual pattern perception as an ade- quate framework with which to explain brightness percepts. For example, the low-frequency spatial components in the two Bergström profiles in Figure 10 are similar, whereas the step-like contour in Figure 10b also contains high-spatial-frequency components. One might therefore

\section{BERGSTRÖM BRIGHTNESS PARADOX (1)}

INPUT PATTERN

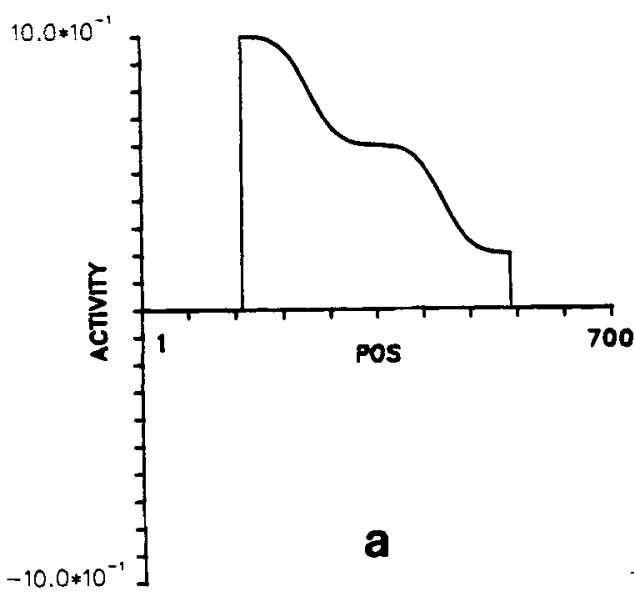

FEATURE CONTOUR PATTERN

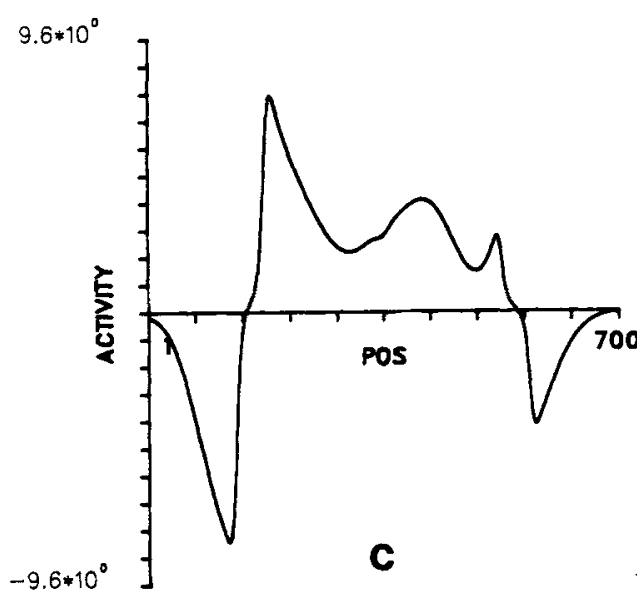

BOUNDARY CONTOUR PATTERN

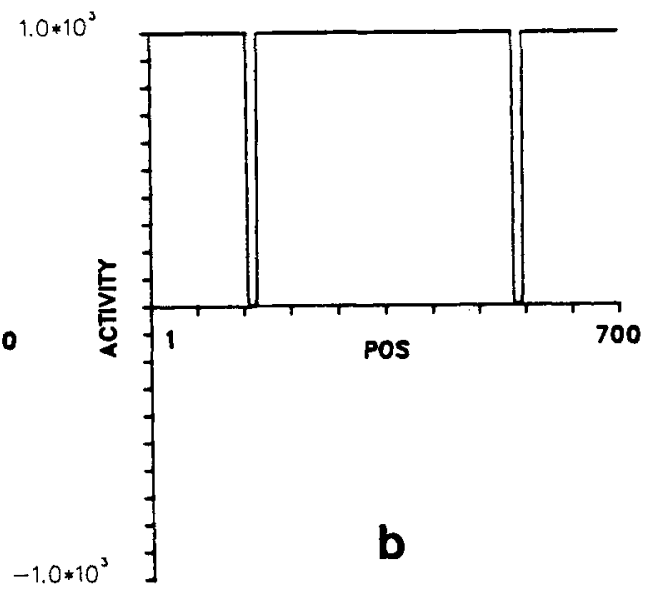

MONOCULAR BRIGHTNESS

PATTERN

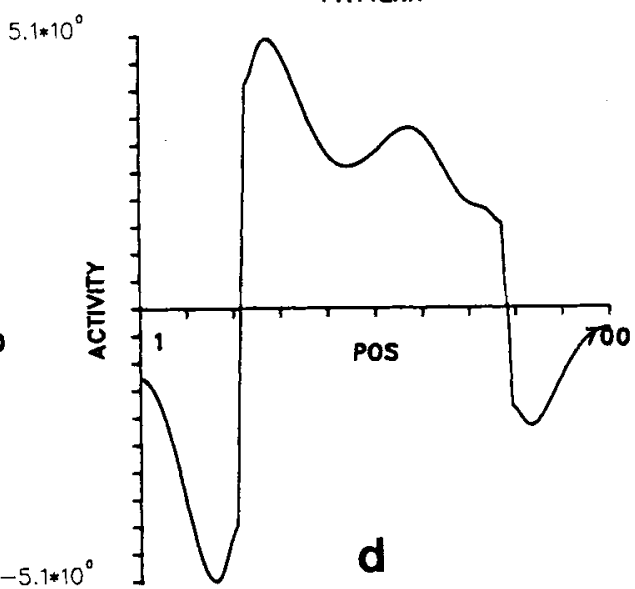

Figure 12. Simulation of a Bergström (1960) brightness experiment. The input pattern (a) generates boundary contours in (b) only around the luminance profile as a whole. By contrast, the input pattern in Figure 13a generates boundary contours around each step in luminance (Figure 13b). The input patterns in Figures 12a and 13a thus determine different syncytial domains within which featural filling-in can occur. The input patterns in Figures $12 \mathrm{a}$ and $13 \mathrm{a}$ also determine different feature-contour patterns (Figures 12c and 13c). The feature-contour pattern in Figure $13 \mathrm{c}$ is more active at position $x_{3}^{*}$ than is the feature-contour pattern of Figure 12c at the corresponding position $x_{3}$. (See Figure 10 for definitions of $x_{3}$ and $x_{3}^{*}$.) The feature-contour pattern of Figure $12 \mathrm{c}$ diffuses within the syncytial domains of Figure 12b, and the featurecontour pattern of Figure 13c diffuses within the syncytial domains of Figure 13b. The resultant brightness pattern of Figure 12d is more active at position $x_{3}$ than is the brightness pattern of Figure 13d at position $x_{3}^{*}$. This feature-to-brightness reversal is due to the fact that the boundary-contour patterns and feature-contour patterns induced by the two input patterns are different. The global structuring of each feature-contour pattern within each syncytial domain determines the ultimate brightness pattern. 


\section{BERGSTRÖM BRIGHTNESS PARADOX (2)}
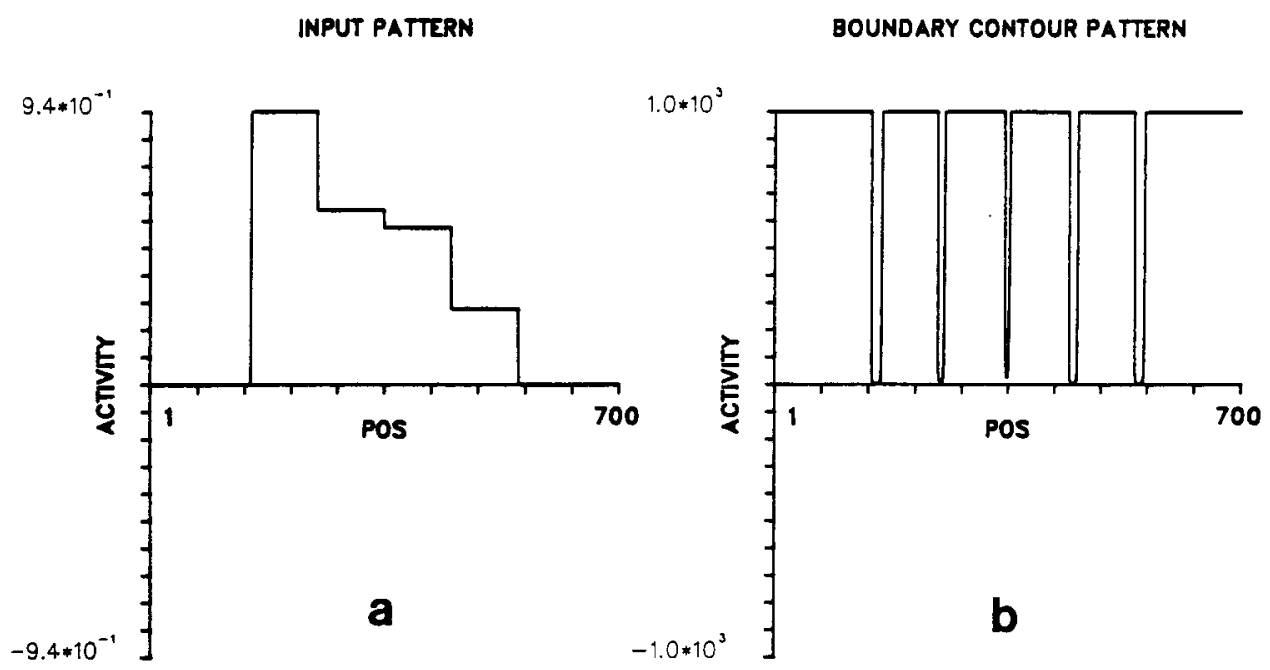

FEATURE CONTOUR PATTERN

MONOCULAR QRIGHTNESS
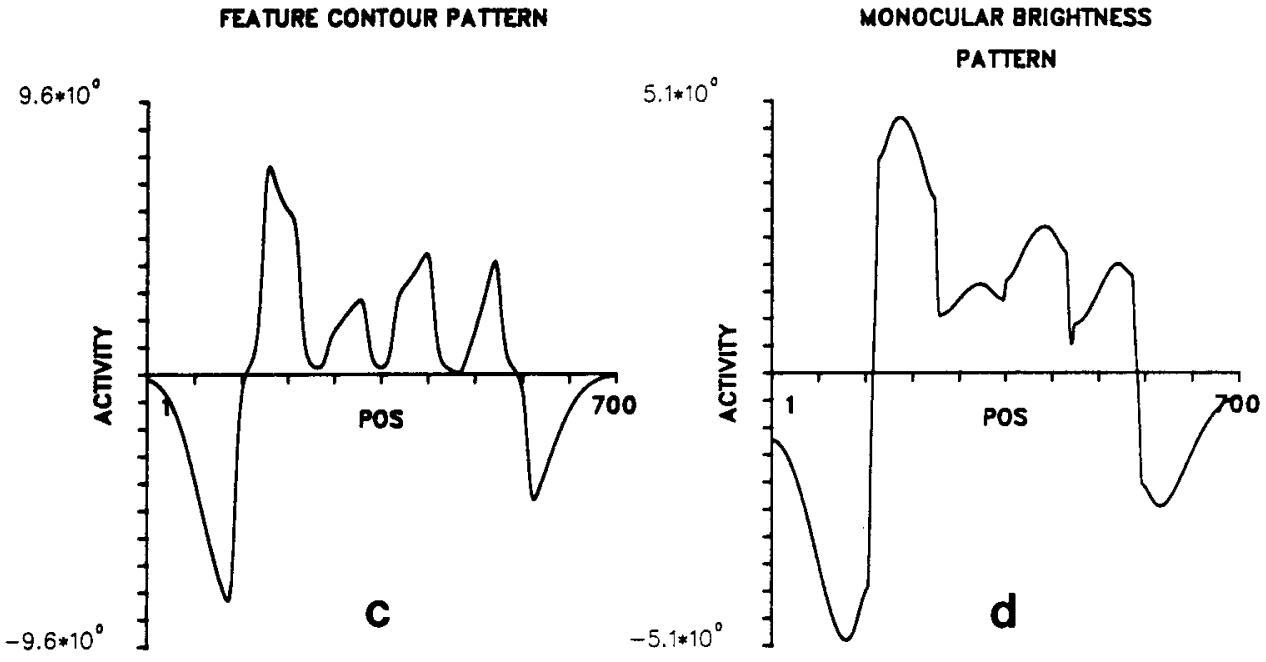

Figure 13. Simulation of a Bergström (1966) brightness experiment. See caption of Figure 12.

expect position $\mathrm{x}_{3}$ to look brighter than position $\mathrm{x}_{3}^{*}$, whereas the reverse is true. In a similar fashion, when a rectangular luminance profile is Fourier analyzed using the human modulation transfer function (MTF), it comes out looking like a Craik-O'Brien contour (Cornsweet, 1970). A Craik-O'Brien contour also comes out looking like a Craik-O'Brien contour. Our explanation, by contrast, shows why both Craik-O'Brien contours and rectangular contours look rectangular.

Some advocates of the Fourier approach have responded to this embarrassment by saying that what the outputs of the MTF look like is irrelevant, since only the identity of these outputs is of interest. This argument has carefully selected its data. It does not deal with the problem that the interior and exterior activities of a Craik-O'Brien contour are the same and differ from the activities of the cusp boundary, whereas the interior and boundary activities of a rectangle are the same and differ from the activities of the rectangle exterior. The problem is not merely one of the equivalence between two patterns. It is also one of the recognition of an individual pattern. These difficulties of the Fourier approach do not imply that multiple spatial scales are unimportant during visual pattern perception. Multiple scale processing does not, however, provide a complete explanation. Moreover, the feature-contour processing within each scale needs to use shunting interactions, rather than the additive interactions of the Fourier theory, in order to extract the relative con- 
trasts of the feature-contour pattern (Appendix A and B; Grossberg, 1983b).

\section{(11) The Assymmetry between Brightness \\ Contrast and Darkness Contrast}

In the absence of a theory to explain the Arend et al. and Bergström data, one might have hoped that a more classical explanation of these effects could be discovered by a more sophisticated analysis of the role of contrast enhancement in brightness perception. In both paradigms, it might at first seem that contrast enhancement around edges or inflection points could explain both phenomena in a unified way, if only a proper definition of contrast enhancement could be found. The following data of Hamada (1980) indicate, in a particularly vivid way, that more than a proper definition of contrast enhancement is needed to explain brightness data.

Figure 14 depicts three luminance profiles. Figure 14a, a uniform background luminance is depicted. (Although the background luminance is uniform, it is not, strictly speaking, a ganzfeld, for it is viewed within a perceptual frame.) In Figure 14b, a brighter Craik-O'Brien luminance profile is added to the backgound luminance. In Figure 14c, a darker Craik-O'Brien luminance profile is subtracted from the background luminance. The purity of this paradigm derives from the facts that its two CraikO'Brien displays are equally long and that the background
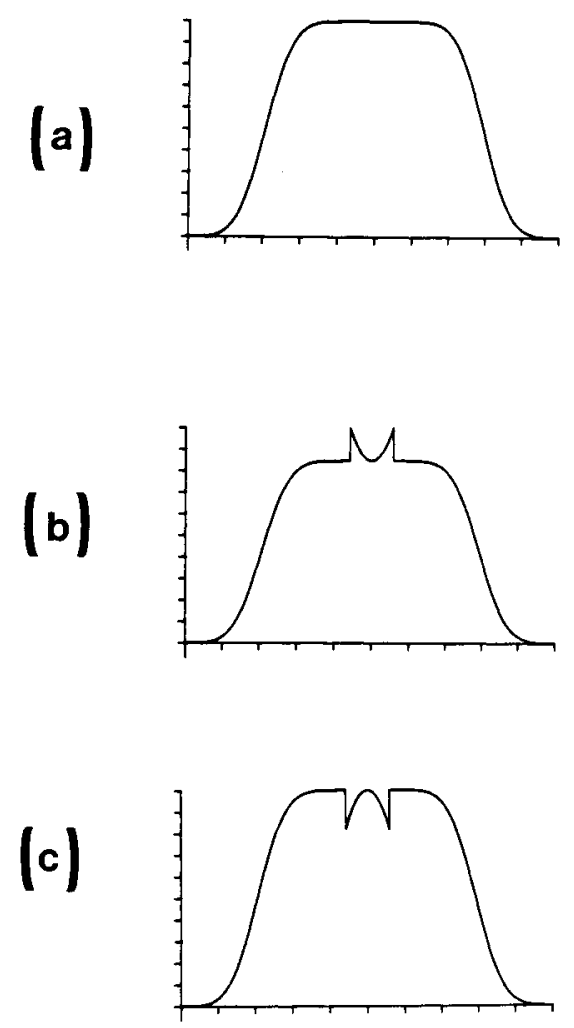

Figure 14. The luminance contours studied by Hamada (1980). All backgrounds in (a)-(c) have the same luminance. luminance is constant in all the displays. Thus, brightening and darkening effects can be studied uncontaminated by other variables.

The classical theory of brightness contrast predicts that the more luminous edges in Figure 14b will look brighter than the background in Figure 14a and that, due to brightness contrast, the background around the more luminous edges in Figure 14b will look darker than the uniform pattern in Figure 14a. This is, in fact, what Hamada found. The classical theory of brightness contrast also predicts that the less luminous edges in Figure $14 \mathrm{c}$ will look darker than the background in Figure 14a and that, due to brightness contrast, the background around the less luminous edges in Figure 14c will look brighter than the background in Figure 14a. Hamada (1980) found, contrary to classical theory, that both the dark edges and the background in Figure 14c look darker than the background in Figure 14a. These data are paradoxical because they show that brighter edges and darker edges are, in some sense, asymmetrically processed, with brighter edges eliciting less paradoxical brightness effects than darker edges.

Hamada $(1976,1978)$ developed a multistage mathematical model to attempt to deal with his challenging data. This model is remarkable for its clear recognition that a "nonopponent"' type of brightness processing is needed in addition to a contrastive, or edge-extracting, type of brightness processing. Hamada did not define boundary contours or diffusive filling-in between these contours, but his important model should nonetheless be better known.

Figures 15 and 16 depict a simulation of the Hamada data using our theory. As desired, classical brightness contrast occurs in Figure 15, whereas as nonclassical darkening of both figure and ground occurs in Figure 16. The dual action of signals from the BCS stage to the MBC stages as boundary-contour signals and as inhibitory feature-contour signals contributes to this result in our simulations.

All of the results described up to now consider how activity patterns are generated within the $M B C_{L}$ and $M B C_{R}$ stages. In order to be perceived, these patterns must activate the BP stage. In the experiments already discussed, the transfer of patterned activity to the BP stage does not introduce any serious constraints on the brightness properties of the FIRE model. This is because all the experiments that we have thus far considered present the same image to both eyes. The experiments that we now discuss present different combinations of images to the two eyes. Thus they directly probe the process whereby monocular brightness domains interact to generate a binocular brightness percept.

\section{(12) Simulations of FIRE}

In the remaining sections of the article, we describe computer simulations using the simplest version of the FIRE process and the same model parameters that were used in Cohen and Grossberg (1984). We show that this model qualitatively reproduces the main properties of 


\section{HAMADA BRIGHTNESS PARADOX (1)}

INPUT PATTERN

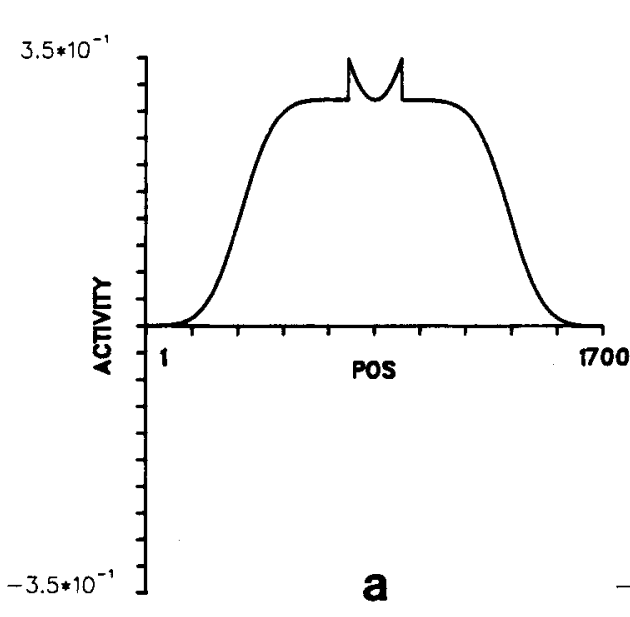

FEATURE CONTOUR PATTERN

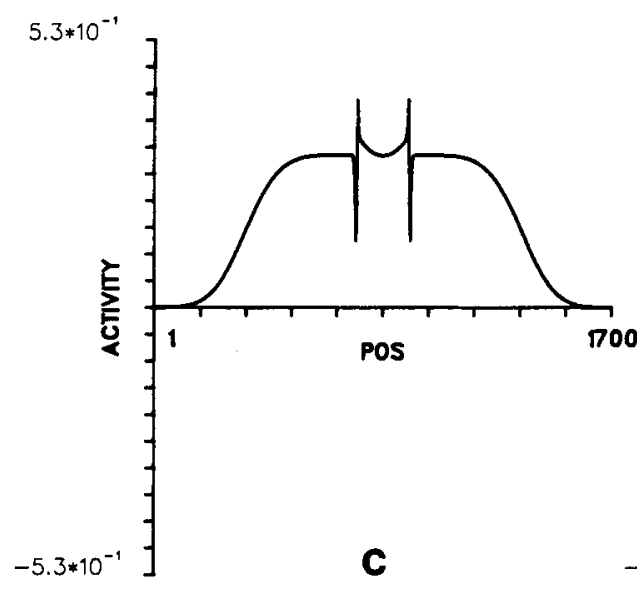

BOUNDARY CONTOUR PATTERN

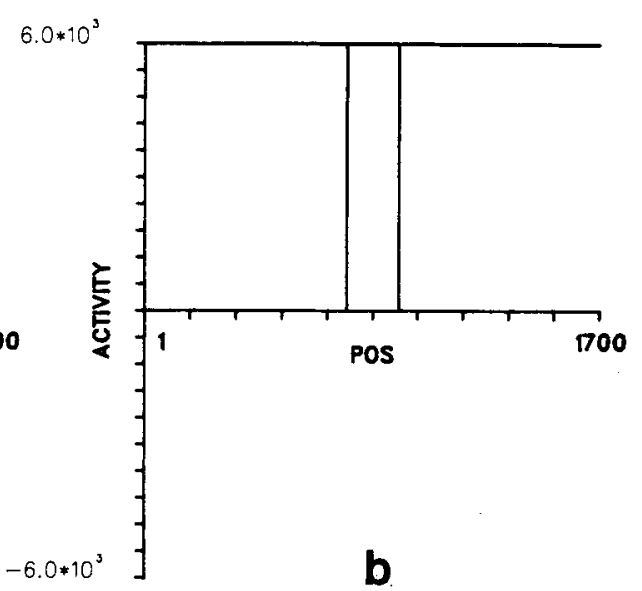

MONOCULAR BRIGHTNESS

PATTERN

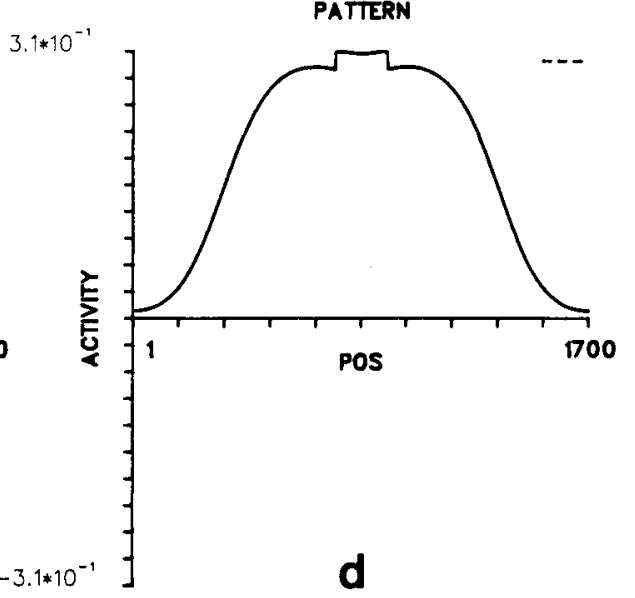

Figure 15. Simulation of the Hamada (1980) brightness experiment. The dotted line in (d) describes the brightness level of the background in Figure 13a. Classical contrast enhancement is obtained in (d).

Fechner's paradox (Levelt, 1965), binocular brightness summation and averaging (Blake, Sloane, \& Fox, 1981; Curtis \& Rule, 1980), and a parametric brightness study of Cogan (1982) on the effects of rivalry, nonrivalry suppression, fusion, and contour-free images. Thus, although the model was not constructed to simulate these brightness data and does not incorporate many known theoretical refinements, it performs in a manner that closely resembles difficult data. We believe that these simulations place the following quotation from a recent publication into a new perspective: "The emerging picture is not simple ... Levelt's theory ... works for binocular brightness perception, but not for sensitivity to a contrast probe ... it seems unlikely that any single mechanism can account for binocular interactions ... The theory of binocular vision is essentially incomplete" (Cogan, 1982, pp. 14-15).

Before reporting simulations of brightness experiments, we review a few basic properties of this FIRE model. All the simulations were done on one-dimensional arrays of cells, for simplicity. All the simulations use pairs of input patterns that have zero disparity with respect to each other. The reaction of a single spatial scale to these input patterns will be reported. Effects of using nonzero disparities and multiple spatial scales are described in Cohen and Grossberg (1984) and Grossberg (1983b). The input patterns should be interpreted as monocular patterns across $\mathrm{MBC}_{\mathbf{L}}$ and $M B C_{R}$, rather than the scenic images themselves.

(a) Insensitivity to functional ganzfelds. In Figure 17, two identical input patterns exist at the $M B C_{L}$ and $M B C_{R}$ 


\section{HAMADA BRIGHTNESS PARADOX (2)}

INPUT PATTERN

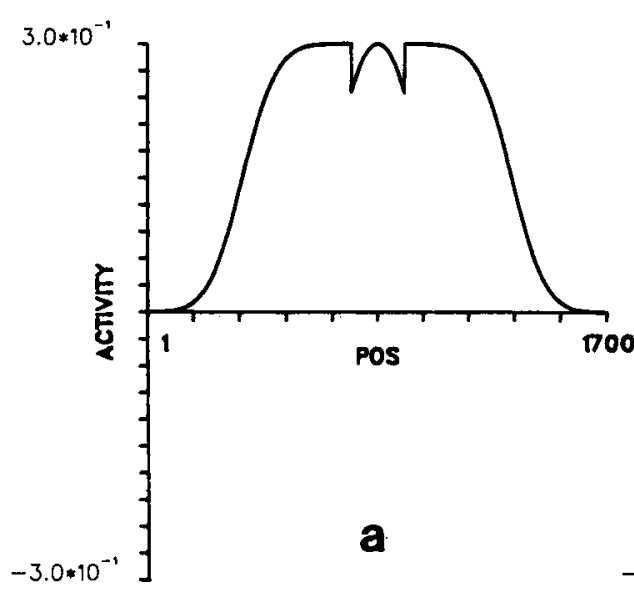

FEATURE CONTOUR PATTERN

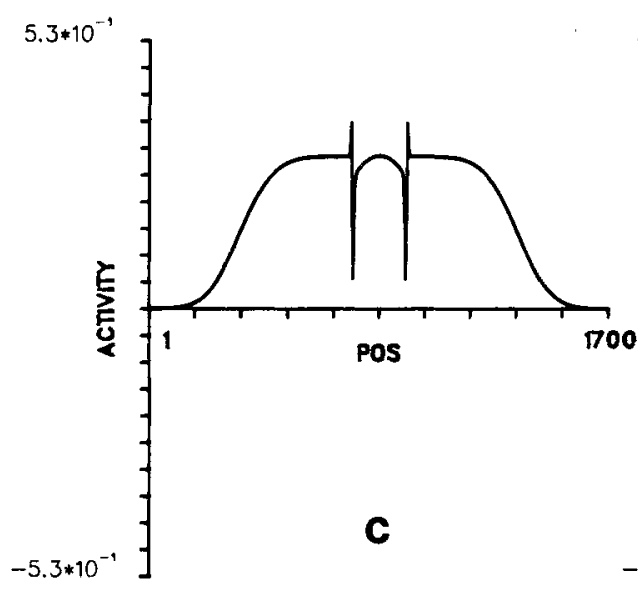

BOUNDARY CONTOUR PATTERN

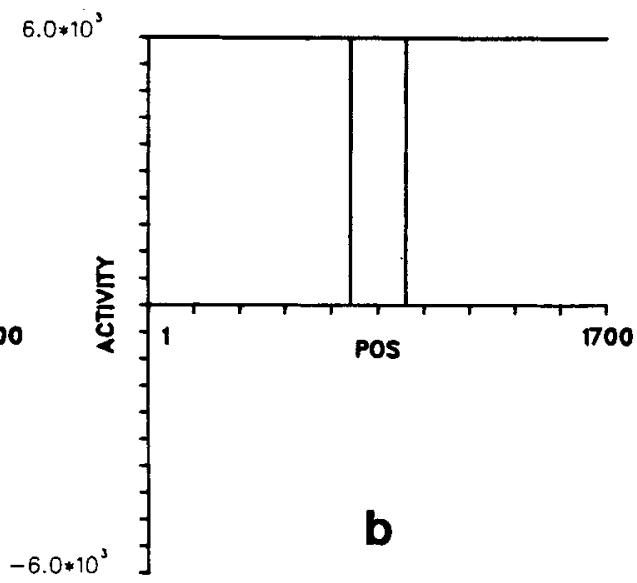

MONOCULAR BRIGHTNESS

PATTERN

$3.1 * 10^{-1}$

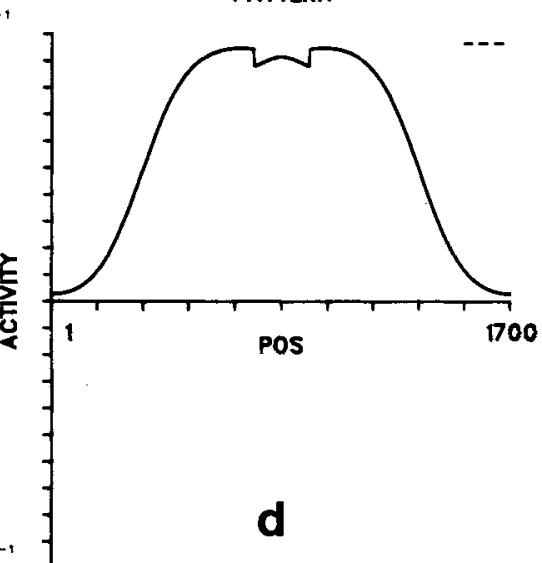

Figure 16. Simulation of the Hamada (1980) brightness experiment. The dotted line in (d) describes the brightness level of the background in Figure 13a. Both background and cusp of (a) look darker than this reference level.

stages (Figure 17a). Both input patterns are generated by putting a rectangular pattern through a Gaussian filter. This smoothing operation was sufficient to prevent the pathways $\mathrm{MBC}_{\mathrm{L}} \rightarrow \mathrm{BP}$ and $\mathrm{MBC}_{\mathrm{R}} \rightarrow \mathrm{BP}$ in Figure 4 from detecting suprathreshold contours in the input patterns. We call an input pattern that has no contours that are detectable by these pathways a "functional ganzfeld." The FIRE process does not lift functional ganzfelds at any input intensity. The simulation illustrates that the BP stage is insensitive to input patterns that include no boundary contours detectable by its filtering operations.

(b) Figure-ground synthesis: Ratio scale and power law. Figure 18 describes the FIRE reaction that is triggered when a rectangular input pattern is superimposed upon a functional ganzfeld. Such an input pattern ideal- izes a region of rapid change in activity with respect to the network's filter bandwidth. The entire input pattern is now resonantly lifted into the BP stage. Although the BP stage is totally insensitive to the functional ganzfeld taken in isolation, the sharp edges of the rectangle trigger a resonant reaction that structures, indeed defines, the functional ganzfeld as a "ground" for the rectangular "figure." Instead of being treated as merely formless energy, the functional ganzfeld now energizes a standing wave that propagates from the rectangle edges to the perimeter of the pattern.

Due to the rectangle's edges, the network is now exquisitely sensitive to the ratio of rectangle-to-ganzfeld input activities. When the entire input pattern is parametrically increased by a common multiple, FIRE activity levels 

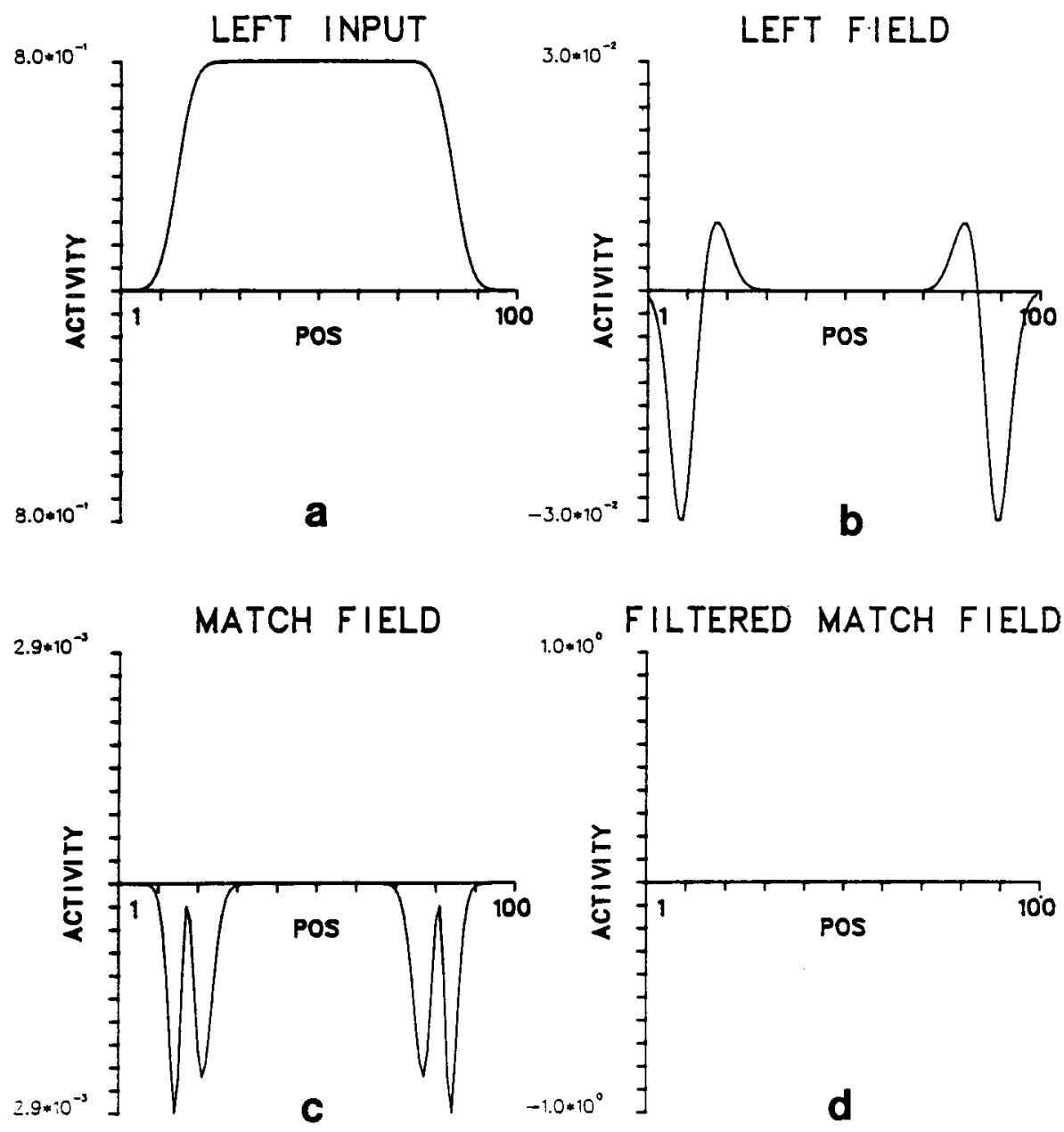

Figure 17. Matched ganzfelds in (a) cause no suprathreshold reaction at the BP stage at any input intensity. Left input in (a) denotes the input pattern that is delivered to both the MP $\mathrm{P}_{\mathrm{L}}$ stage and the $M P_{R}$ stage. Left field in (b) denotes the activity pattern that is elicited at both the $M C_{L}$ stage and the $M_{B C}$ stage. Match field in (c) denotes the activity pattern that is elicited at the $B P$ stage. Filtered match field in (d) denotes the feedback signal pattern that is emitted from the BP stage to both the $M_{B C}$ and $M B C_{R}$ stages. No feedback is elicited because the BP stage does not generate any suprathreshold activities in response to the edgeless input pattern, or functional ganzfeld, in (a). (Reprinted from Cohen \& Grossberg, 1984.)

obey a power law (Figure 19). Both the intensity of the standing wave corresponding to the rectangle and the intensity of the standing wave corresponding to the functional ganzfeld grow as a power of their corresponding input intensities. In these simulations, the power approximates .8. This power is not built into the network. It is a collective property of the network as a whole.

\section{(13) Fechner's Paradox}

The simplest version of Fechner's paradox notes that the world does not look half as bright when one eye is closed. In fact, suppose that a scene is viewed through both eyes but that one eye sees it through a neutral density filter (Hering, 1964). When the filtered eye is entirely occluded, the scene looks brighter and more vivid despite the fact that less total light reaches the two eyes.

Another version of this paradox is described in
Figure 20 (Cogan, 1982; Levelt, 1965). Figures 20a-20c depict three pairs of images. One image is viewed by each eye. In Figure 20a, an uncontoured image is viewed by the left eye and a black disk on a uniform background is viewed by the right eye. In Figure 20b, black disks are viewed by both eyes. In Figure 20c, the interior of the left disk is white. Given appropriate boundary conditions, the binocular percept generated by the images in Figure 20a looks about as dark as the binocular percept generated by the images in Figure 20b, despite the fact that a bright region in Figure 20a replaces a black disk in Figure 20b. Figure 20c, by contrast, looks much brighter.

The input patterns that we used to simulate these images are displayed in Figures $20 \mathrm{~d}-20 \mathrm{~g}$. These input patterns represent the images in only a crude way, because the input patterns correspond to activity patterns across stages $M B C_{L}$ and $M B C_{R}$ rather than to the images themselves. 

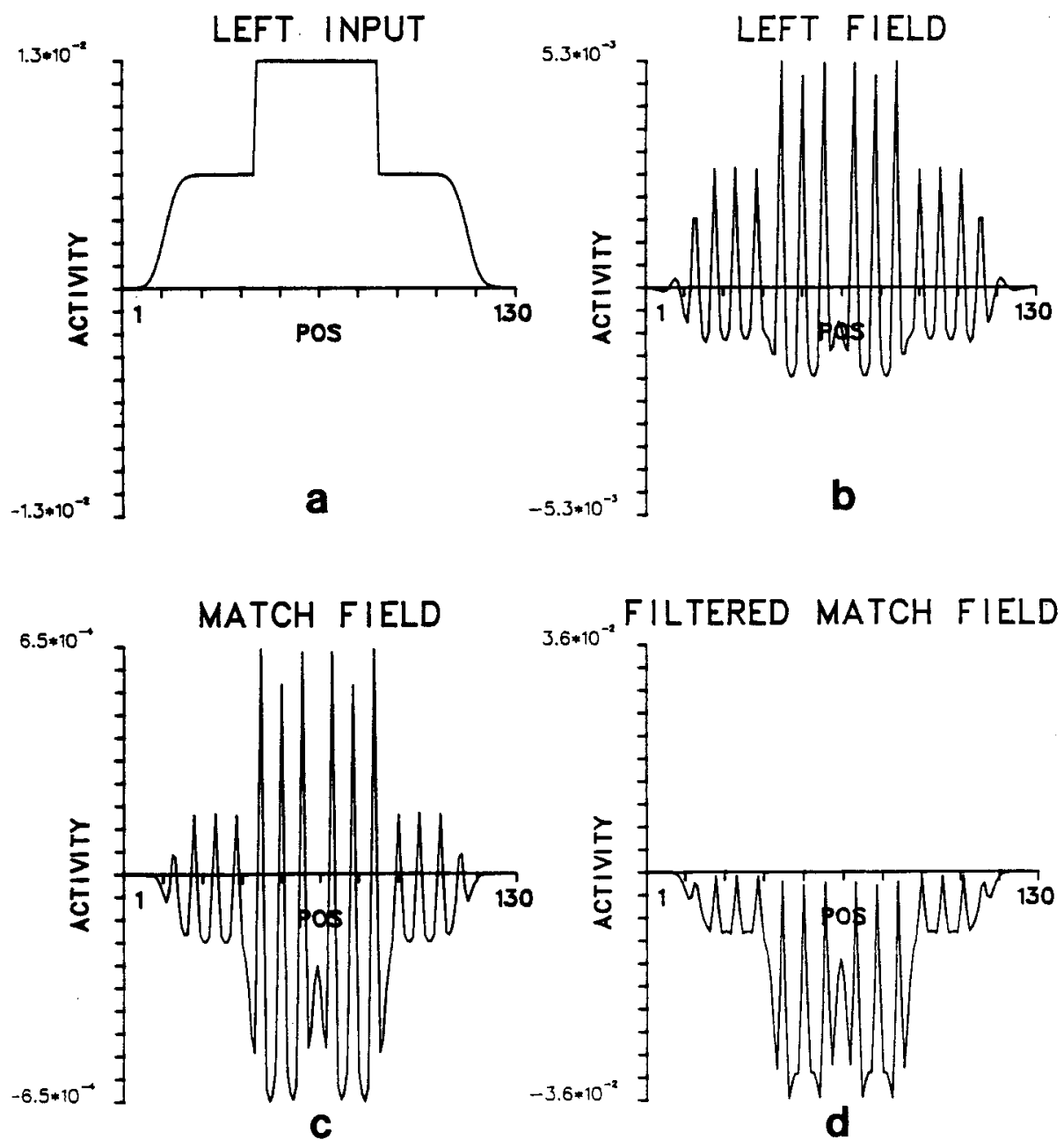

Figure 18. Figure on ganzfeld: The pair of sharp contours within the input pattern of (a) sensitizes the BP stage to the activity levels of both the rectangle figure and the ground, despite the total insensitivity of the BP stage to a functional ganzfeld in Figure 17 at any input intensity. Binocular matching of the contours at the BP stage lifts a standing wave representation (c) of figure and ground into the BP stage. (Reprinted from Cohen \& Grossberg, 1984.)

It is uncertain how, for example, to choose the activity of the ganzfeld in Figure 20a, since this activity depends upon the total configuration of contours throughout the field of view. We therefore carried out a simulation using a zero intensity ganzfeld, as well as a simulation with a functional ganzfeld whose intensity equals the background intensity of the input pattern to the the other MBC stage. The actual functional ganzfeld intensity should lie somewhere in between these two values. Other approximations of this type are used throughout the simulations.

The numbers listed in Figures $20 \mathrm{~d}-20 \mathrm{~g}$ describe the total rectified output from the FIRE cells that subtend the region corresponding to the black disk. As in the data, Figure $20 \mathrm{~g}$ generates a much larger output than Figure 20f. Figure $20 \mathrm{~g}$ also generates a larger output than either Figure $20 \mathrm{~d}$ or Figure $20 \mathrm{e}$. If the actual functional ganzfeld level is small due to the absence of nearby feature-contour signals, then Figures 20a and $20 \mathrm{~b}$ will look equally bright to the network.
A comparison between Figures 20d and 20e provides the first evidence of a remarkable formal property of this version of the FIRE model. Although the FIRE process is totally insensitive to a pair of functional ganzfelds, when a functional ganzfeld is binocularly paired with a contoured figure, it can influence the overall intensity of binocular activity within the BP stage.

\section{(14) Binocular Brightness Averaging and Summation}

Experimental studies of the conditions under which Fechner's paradox hold have led to the conclusion that "binocular brightness should represent a compromise between the monocular brightnesses when the luminances presented to the two eyes are grossly different and ... it should exceed either monocular brightness when their luminances approach equality" (Curtis \& Rule, 1980, p. 264). Curtis and Rule point out that "these results were in conflict with the prediction of averaging models, such 


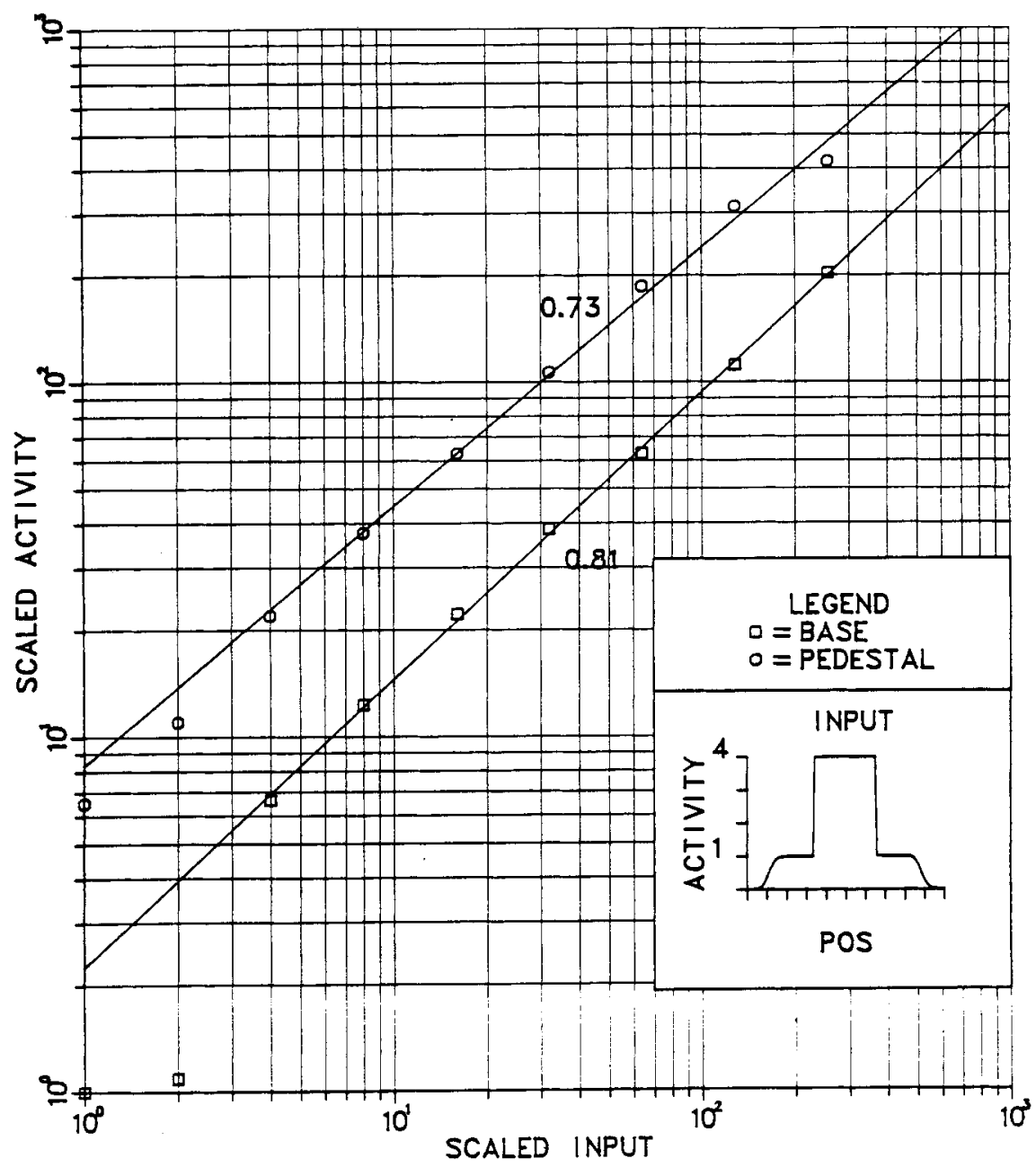

Figure 19. Power-law processing of figure and ground activity levels at the BP stage as the intensities of the input pattern (in the insert) are proportionally increased by a common factor. The abscissa (scaled input) measures this common factor. The ordinate (scaled activity) measures the peaks of BP activity at the rectangle (circles) and the ground (squares). (Reprinted from Cohen \& Grossberg, 1984.)

as those of Engel (1969) and Levelt (1965)"' (p. 263). They introduce a vector model to partially overcome this difficulty. Although the averaging and vector models are useful in organizing brightness data, they do not provide a mechanistic explanation of these data.

Figure 21 describes an example of binocular averaging by the FIRE process. In Figures $21 \mathrm{a}$ and $21 \mathrm{~b}$, one of the input patterns is a functional ganzfeld. The other input pattern is an increment or a decrement on a background. Since these monocular input figures differ greatly in intensity, binocular brightness averaging should occur when they are binocularly presented. In Figure $21 \mathrm{c}$, the increment input pattern is paired with a decrement input pattern. The binocular figural activity in Figure 21c almost exactly equals the average of the monocular figural activities in Figures 21a and $21 \mathrm{~b}$.

In Figure 21d, a pair of increment input patterns is presented to the model. A comparison of Figure $21 \mathrm{~d}$ with
Figure 21a shows that the binocular figural activity in Figure $21 \mathrm{~d}$ is significantly greater than the monocular figural activity in Figure $21 \mathrm{a}$; that is, binocular brightness summation has occurred. Using these inputs, the binocular brightness is about $25 \%$ greater than the monocular brightness. Using a fully attenuated (zero) ganzfeld in one eye during the monocular condition, the binocular brightness is about $63 \%$ brighter than the monocular brightness. Nonlinear binocular summation in which the binocular percept is less than twice as bright as the monocular percept has been described by a number of investigators (Blake et al. 1981; Cogan et al., 1982; Legge \& Rubin, 1981).

\section{(15) Simulation of a Parametric Binocular Brightness Study}

Cogan (1982) has analyzed binocular brightness interactions by studying a subject's sensitivity to monocular 


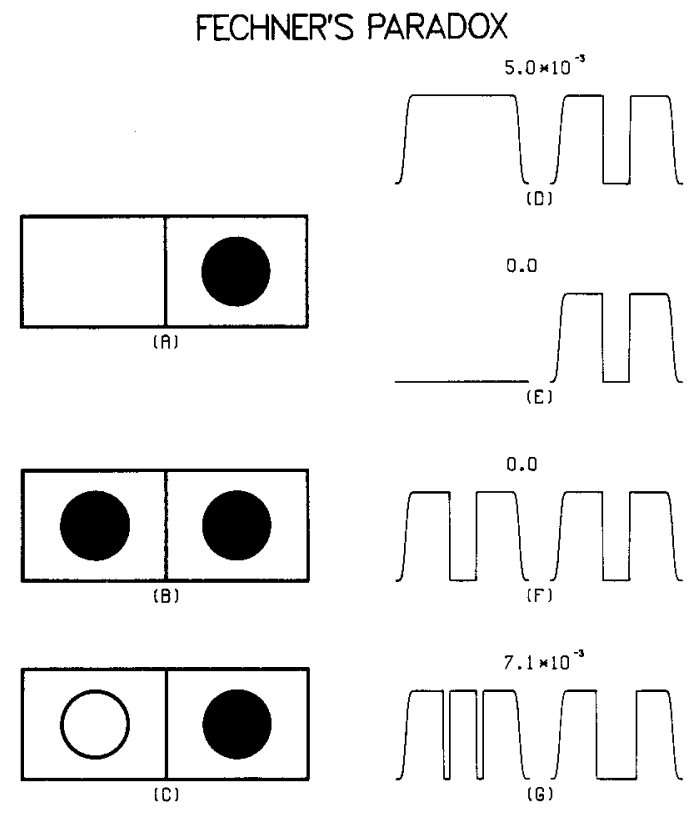

Figure 20. Fechner's paradox: In human experiments based on the images in (a)-(c), the left image is viewed by the left eye while the right image is viewed by the right eye. The simulations used the pairs of patterns in (d)-(g) as left and right input patterns to the FIRE process. Ganzfelds of different intensity are used as left input patterns to the FIRE model in (d) and (e). The FIRE activity levels corresponding to the dark region positions in the right input patterns are printed above. In vivo, the ganzfeld intensity of a large field will be close to zero at the $\mathrm{MBC}_{\mathrm{L}}$ stage, as in (e). In (f), identical left and right input patterns elicit zero FIRE activity in the dark region. In (g), the dark region generates the largest FIRE activity of the series.

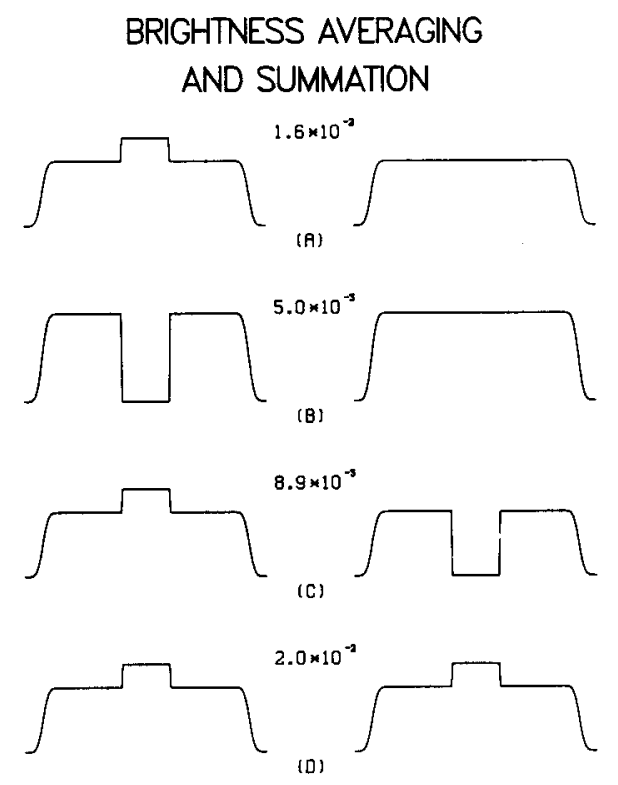

Figure 21. Brightness averaging and summation: The input pair in (c) generates a FIRE activity at their center that is approximately the average of the FIRE activities generated at the center positions of the input pairs in (a) and (b). The input pair in (d) generates a FIRE activity that is greater at its center than the FIRE activity generated at the center of the input pattern in (a). test flashes while the subject binocularly views different pairs of monocular images. Cogan used this method of limits to obtain psychometric curves, and then rankordered paradigms in terms of subject sensitivity. Figure 22 describes the five conditions that Cogan studied in his Experiment 2. In each condition, a brief disk-shaped flash was presented to the left eye. The flash area was chosen to fit exactly within the circular contour in the left image. Figure 23 describes the sensitivity of six different subjects to each of the five pairs of images. Mean detection sensitivity tended to rank-order the images from Figure 22a to Figure 22e in order of decreasing sensitivity. Mean sensitivity to the images of Figure 22a was significantly greater than to the other images over a wide range of probe contrasts $(\Delta \mathrm{I} / \mathrm{I})$. Mean sensitivity to Figure $22 \mathrm{e}$ was significantly less than to the other images over a wide range of probe contrasts. Mean sensitivity to the other images grouped more closely together. The rank orderings of individual observers did not, moreover, always decrease from Figure 22b to Figure 22d.

Simulations using the simplest one-dimensional input versions of the images in Figure 22 tended to reproduce this pattern of results. Figure 24 illustrates the input pairs that were used. Each input pair represents the flash condition. The increment above the background level on the left input pattern represents the flash. To estimate flash visibility, we first computed the figural activity within the flash area that was generated before the flash, then computed the figural activity within the flash area that was generated during the flash, and then subtracted the beforeflash activity from the after-flash activity. The before-flash

\section{FLASH DISPLAYS}
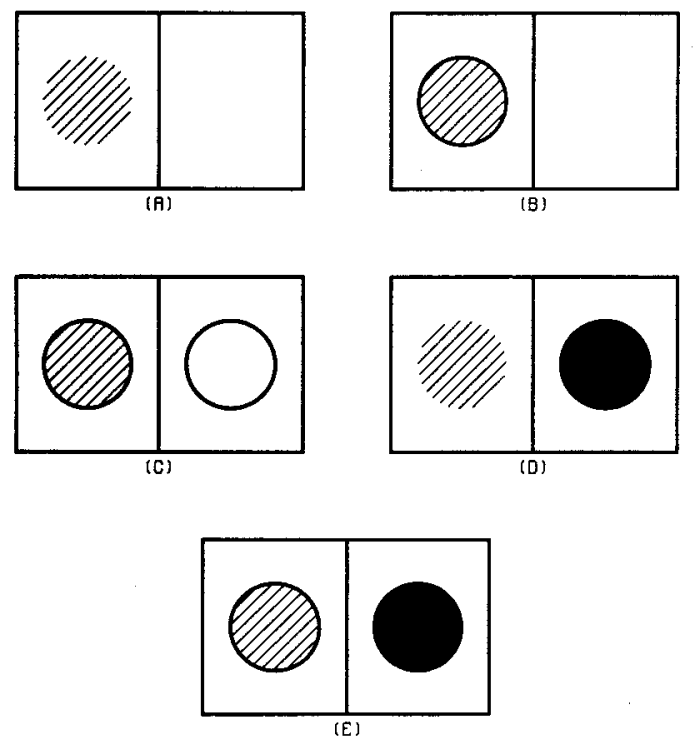

Figure 22. Flash displays used by Cogan (1982) to study binocular brightness processing. The dashed lines denote the regions that receive monocular flashes. Cogan tested the sensitivity of subjects to flashes in the designated positions. 

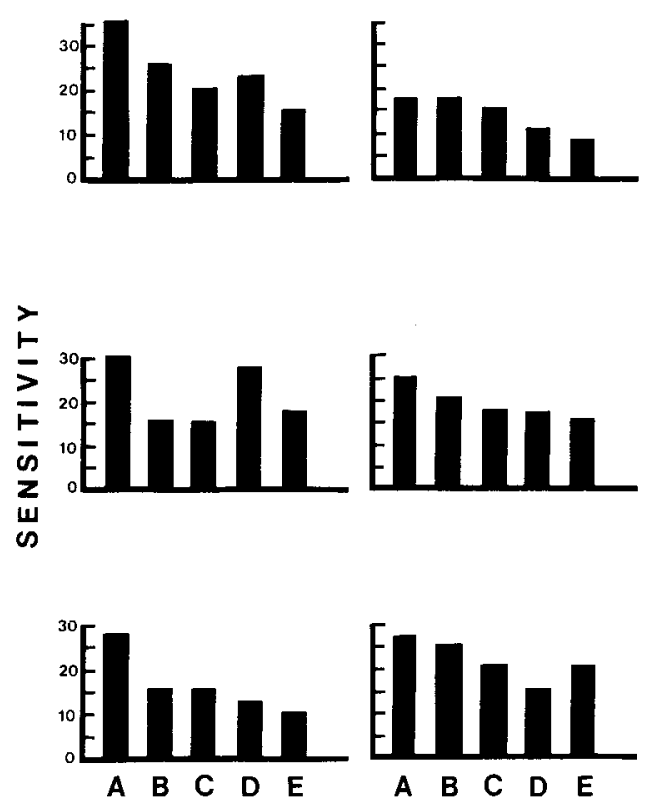

Figure 23. Sensitivity of individual subjects to the flash displays described in Figure 22. Each bar height corresponds to a subject's sensitivity to a particular flash display. The labels (A)-(E) refer to the flash conditions in Figure 22. (Redrawn from Cogan, 1982.)

\section{FLASH PROFILES}
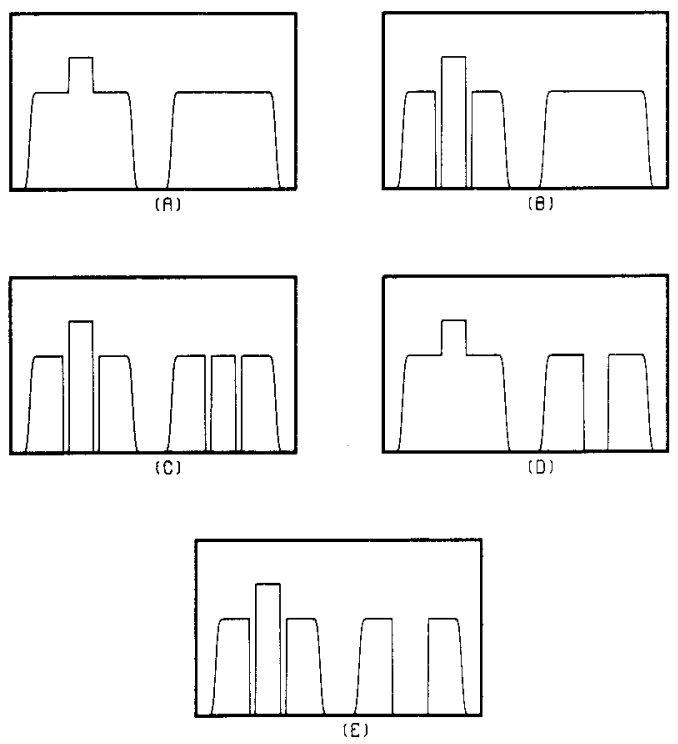

Figure 24. Flash profiles used to simulate the Cogan (1982) experiment. These profiles depict the profile when the flash is on. Before the flash is on, all the increments above the background luminance are absent.

activities, after-flash activities, and flash-induced activity differences are listed in Table 2. As in the data, the images in Figure 24a generated the largest increment, those in Figure $24 \mathrm{e}$ generated smallest increment, and the other three increments were clustered together. The main dis- crepancy with the data is due to the fact that sensitivity to the images in Figure 24d slightly exceeds that to the images in Figures 24c and 24b. This type of order inversion also occurred, however, in two out of six of Cogan's subjects (Cogan, 1982, Figure 6, p. 11). Considering the simplicity of the model and its input patterns, and the number of qualitatively correct effects that it can generate, this seems to be a relatively minor point.

Figure 25 displays the resonant patterns that are generated by four pairs of distinct monocular images. Figures $25 \mathrm{a}$ and $25 \mathrm{~b}$ illustrate the computer experiment in Figure 24d. In Figure 25a, a ganzfeld is paired with a black disk. Although the network is insensitive to a pair of ganzfelds (Figure 17), the black disk at the $M B C_{R}$ stage structures and energizes the ganzfeld at the $M B C_{L}$ stage via the BP stage. The structured ganzfeld, in turn, modifies the activity level at the BP stage. The monocular $\mathrm{MBC}_{\mathrm{R}}$ pattern remains inactive at cells that receive the black input, despite the fact that the binocular FIRE pattern is active within the corresponding region due to the influence of the ganzfeld. Figure $25 \mathrm{~b}$ adds an increment, or flash, to the ganzfeld in Figure 25a. Again, the $M B C_{R}$ pattern remains inactive at cells that receive the black input. A comparison of Figures $25 \mathrm{a}$ and $25 \mathrm{~b}$ shows, however, that the BP stage is sensitive to the activity levels of both monocular patterns within this region. In fact, the activity level in this region of the BP stage in Figure 25b averages the corresponding monocular activities.

Figures 26a and 26b illustrate the computer experiment in Figure $24 \mathrm{e}$. Note that the dark contour in the $\mathrm{MBC}_{\mathrm{L}}$ input pattern is detected by the resonance. This contour monocularly energizes the binocular resonance in Figure 26a much more than does the ganzfeld of equal background intensity in Figure 25a.

\section{(16) Concluding Remarks}

The results in this article suggest that several of the most basic concepts of visual theory need to be refined. For example, the simulations described above include at least three mechanistically different concepts of contour: boundary contour, feature contour, and FIRE contour. They also include two different types of filling-in: diffusive filling-in, which is monocular, and resonant fillingin, which is binocular. Although these concepts add some complexity to the visual modeling literature, they have begun to simplify and unify the explanation of a large body of visual data. The same concepts have been used, for example, to suggest explanations of data concerning monocular and binocular rivalry, illusory figures, fading

Table 2

Simulation of Brightness Experiment in Figure 24

\begin{tabular}{cccc}
\hline Figure & $\begin{array}{c}\text { Activity of } \\
\text { Inner Region } \\
\text { Before Flash }\end{array}$ & $\begin{array}{c}\text { Activity of } \\
\text { Inner Region } \\
\text { During Flash }\end{array}$ & $\begin{array}{c}\text { Activity } \\
\text { Increment }\end{array}$ \\
\hline $24 \mathrm{a}$ & .000000 & .015740 & .015740 \\
$24 \mathrm{~b}$ & .012356 & .016165 & .003809 \\
$24 \mathrm{c}$ & .011824 & .015689 & .003865 \\
$24 \mathrm{~d}$ & .005031 & .008904 & .003873 \\
$24 \mathrm{e}$ & .007075 & .010407 & .003332 \\
\hline
\end{tabular}


(a) GANZFELD PLUS DARK FGGURE
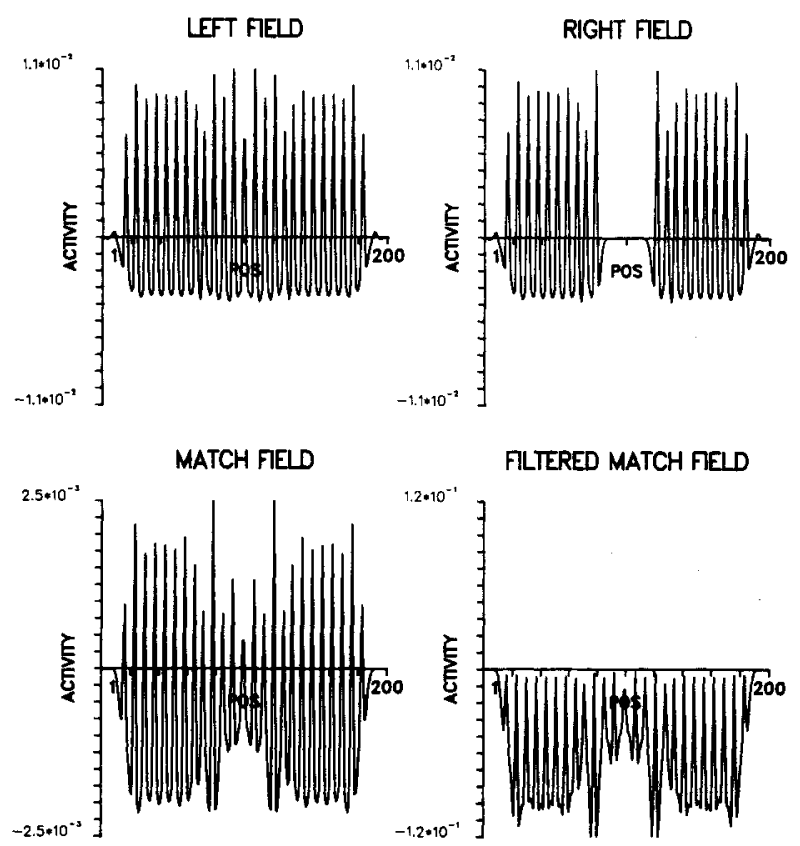

(b) INCREMENT PLUS DARK FIGURE
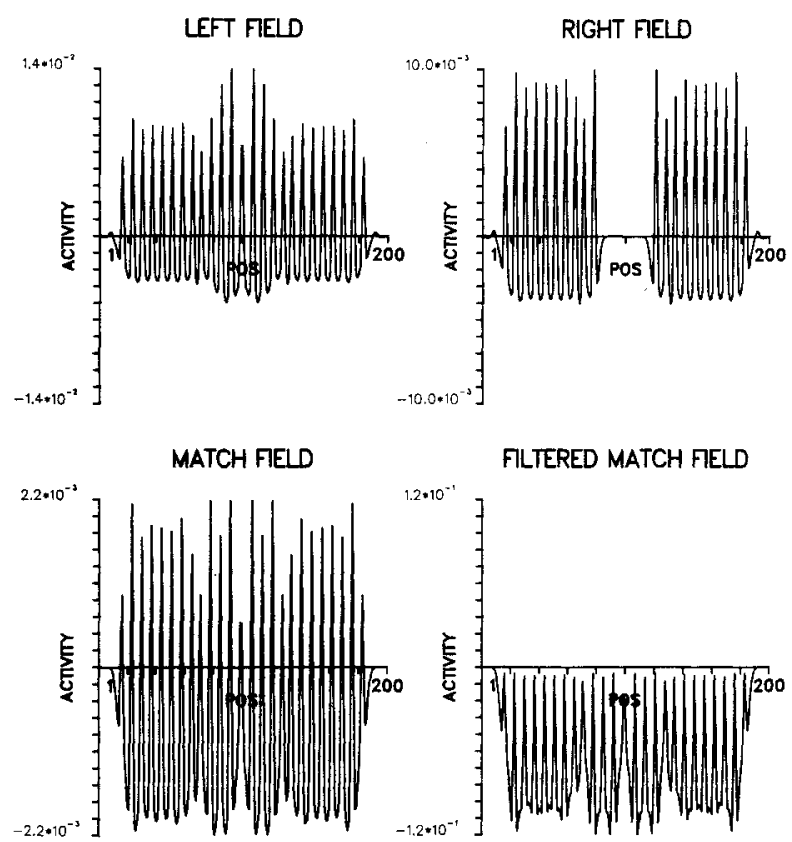

Figure 25. FIRE patterns generated by the flash displays of Figure 24d; (a) Before flash FIRE pattern; (b) during flash FIRE pattern. Left field denotes the $\mathrm{MBC}_{L}$-stage activity pattern. Right field denotes the $\mathrm{MBC}_{\mathrm{R}}$-stage activity pattern. Match field denotes the $B P$-stage activity pattern. Filtered match field denotes the feedback signal pattern emitted by the $B P$ stage to the $M B C_{L}$ and $M B C_{R}$ stages.

(a) BOUNDARY PLUS DARK FIGURE

LEFT FELD

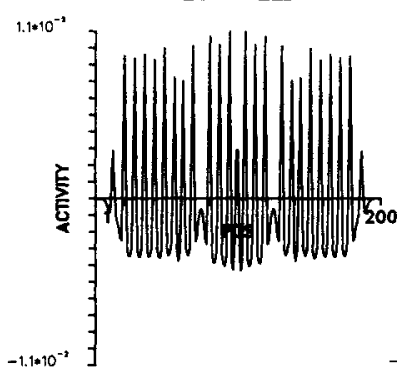

MATCH FELD

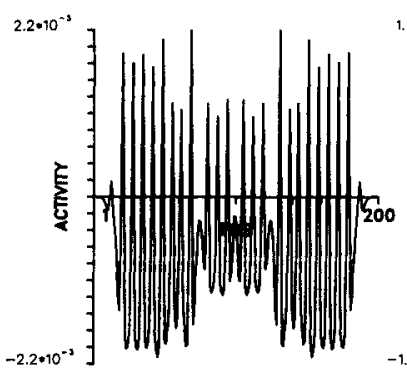

RIGHT FIELD

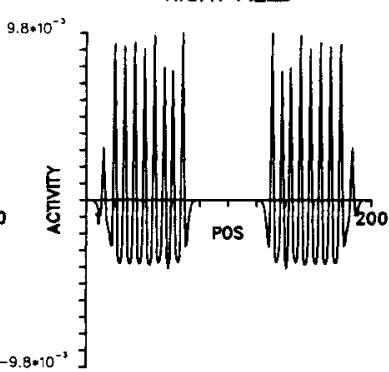

FLTERED MATCH FELD

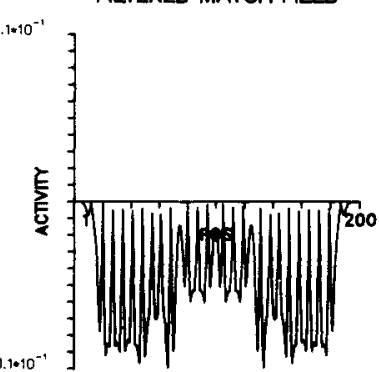

(b)

INCREMENT IN BOUNDARY

LFFT FELL
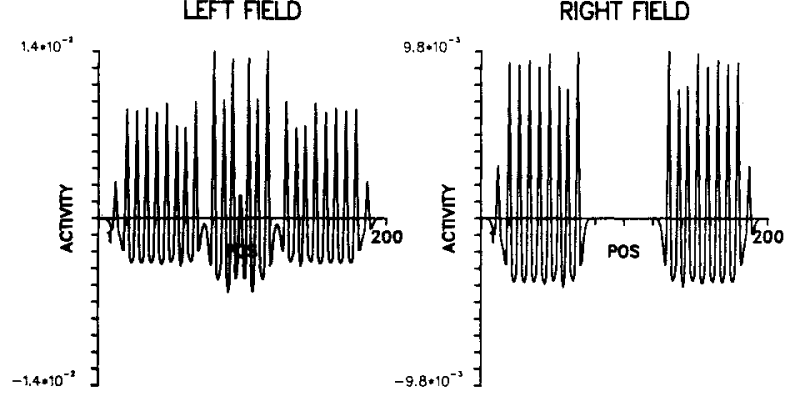

MATCH FELD

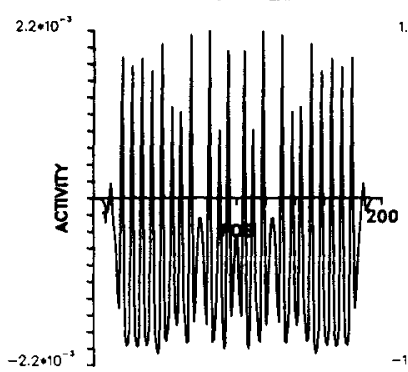

FILTERED MATCH FIELD

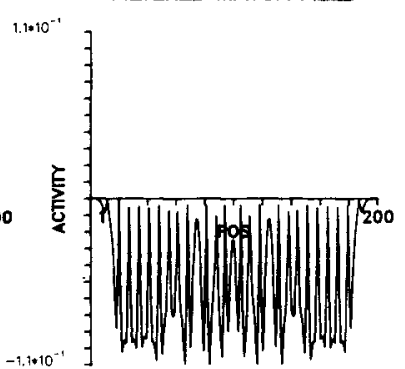

Figure 26. FIRE patterns generated by the flash display of Figure 24e. (a) Before flash FIRE pattern; (b) during flash FIRE pattern. 
of stabilized images, neon color spreading, illusory complementary color induction, the Land retinex demonstrations, nonlinear multiple scale interactions, and various global interactions between depth, lightness, length, and form properties (Cohen \& Grossberg, 1984; Grossberg, 1980, 1983b, 1985; Grossberg \& Mingolla, in press). Moreover, the concepts seem to have more than a formal existence. Boundary contour and feature contour interactions can, for example, be interpreted in terms of recent physiological data concerning the orientationsensitive but color-insensitive hypercolumn system in the striate cortex and the orientation-insensitive but colorsensitive blob system in the striate cortex (Table 1; Grossberg \& Mingolla, in press). It remains to be seen just how far these new concepts and mechanisms can be developed for the further explanation and prediction of complex visual phenomena.

\section{REFERENCES}

Arend, L. E., Buehler, J. N., \& Lockhead, G. R. (1971). Difference information in brightness perception. Perception \& Psychophysics, 9, 367-370

BERGSTRÖM, S. S. (1966). A paradox in the perception of luminance gradients, I. Scandinavian Journal of Psychology, 7, 209-224.

BergSTRöm, S. S. (1967a). A paradox in the perception of luminance gradients, II. Scandinavian Journal of Psychology, 8, 25-32.

BERGSTRÖM, S. S. (1967b). A paradox in the perception of luminance gradients, III. Scandinavian Journal of Psychology, 8, 33-37.

BLAKE, R., \& Fox, R. (1974). Binocular rivalry suppression: Insensitive to spatial frequency and orientation change. Vision Research, 14, 687-692.

Blake, R., Sloane, M., \& Fox, R. (1981). Further developments in binocular summation. Perception \& Psychophysics, 30, 266-276.

CARPENTER, G. A., \& Grossberg, S. (1981). Adaptation and transmitter gating in vertebrate photoreceptors. Journal of Theoretical Neurobiology, 1, 1-42.

Cogan, A. L. (1982). Monocular sensitivity during binocular viewing. Vision Research, 22, 1-16.

Cogan, A. L., Silverman, G. \& Sekuler, R. (1982). Binocular summation in detection of contrast flashes. Perception \& Psychophysics, 31, 330-338.

Cohen, M. A., \& GrossberG, S. (1984). Some global properties of binocular resonances: Disparity matching, filling-in, and figure-ground synthesis. In P. Dodwell \& T. Caelli (Eds.), Figural synthesis. Hillsdale, NJ: Erlbaum.

CoReN, S. (1983). When "filling-in" fails. The Behavioral and Brain Sciences, 6, 661-662.

CoRnsweet, T. N. (1970). Visual perception. New York: Academic Press.

CurTis, D. W., \& RULE, S. J. (1980). Fechner's paradox reflects a nonmonotone relation between binocular brightness and luminance Perception \& Psychophysics, 27, 263-266.

DAY, R. H. (1983). Neon color spreading, partially delineated borders, and the formation of illusory contours. Perception \& Psychophysics, 34, $488-490$.

ENGEL, G. R. (1969). The auto-correlation function and binocular brightness mixing. Vision Research, 9, 1111-1130.

Gellatly, A. R. H. (1980). Perception of an illusory triangle with masked inducing figure. Perception, 9, 599-602.

GerRits, H. J. M., dE HAAN, B., \& Vendrick, A. J. H. (1966). Experiments with retinal stabilized images: Relations between the observations and neural data. Vision Research, 6, 427-440.

Gerrits, H. J. M. \& Timmerman, J. G. M. E. N. (1969). The fillingin process in patients with retinal scotomata. Vision Research, 9 , 439-442.
Gerrits, H. J. M., \& VENDRICK, A. J. H. (1970). Simultaneous contrast, filling-in process and information processing in man's visual system. Experimental Brain Research, 11, 411-430.

Graham, N. (1981). The visual system does a crude Fourier analysis of patterns. In S. Grossberg (Ed.), Mathematical psychology and psychophysiology. Providence, RI: American Mathematical Society.

Graham, N., \& NaChmias, J. (1971). Detection of grating patterns containing two spatial frequencies: A test of single-channel and multiplechannel models. Vision Research, 11, 251-259.

GREGORY, R. L. (1966), Eye and brain. New York: McGraw-Hill.

GrossberG, S. (1975). A neural model of attention, reinforcement, and discrimination learning. International Review of Neurobiology, 18, 263-327

Grossberg, S. (1980). How does a brain build a cognitive code? Psychological Review, 87, 1-51.

GrossBerg, S. (1982a). Studies of mind and brain: Neural principles of learning, perception, development, cognition, and motor control. Boston: Reidel Press.

GrossBeRG, S. (1982b). The processing of expected and unexpected events during conditioning and attention: A psychophysiological theory. Psychological Review, 89, 529-572.

GrossBerG, S. (1983a). Neural substrates of binocular form perceptiom: Filtering, matching, diffusion, and resonance. In E. Basar, H. Flohr, H. Haken, \& A. J. Mandell (Eds.), Synergetics of the brain. New York: Springer.

Grossberg, S. (1983b). The quantized geometry of visual space: The coherent computation of depth, form, and lightness. Behavioral and Brain Sciences, 6, 625-692.

Grossberg, S. (1984). Outline of a theory of brightness, color, and form perception. In E. Degreef \& J. van Buggenhaut (Eds.). Trends in mathematical psychology. Amsterdam: North-Holland.

Grossberg, S. (1985). Neural dynamics of depth, brightness, color, and form perception: A predictive synthesis. Manuscript submitted for publication.

GrossberG, S., \& Mingolla, E. (1985). Nonlinear competition, cooperation, and diffusion in the neural dynamics of visual perception. In B. D. Sleeman \& R. J. Jarvis (Eds.), Proceedings of the Dundee conference on ordinary and partial differential equations, 1984. New York: Springer.

GrossberG, S., \& Mingolla, E. (in press). Neural dynamics of form perception: Boundary completion, illusory figures, and neon color spreading. Psychological Review.

HAMADA, J. (1976). A mathematical model for brightness and contour perception. Hokkaido Report of Psychology, HRP-11-76-17.

HaMADA, J. (1978). Antagonistic and non-antagonistic processes for the lightness perception. Hokkaido Behavioral Science Report, HBSR-P-4.

HAMADA, J. (1980, July 6-12). Antagonistic and non-antagonistic processes in the lightness perception. Proceedings of XXII International Congress of Psychology, Leipzig.

Heggelund, P. \& KreKLing, S. (1976). Edge dependent lightness distributions at different adaptation levels. Vision Research, 16, 493-496.

Helmholtz, H. von (1962). Physiological optics. (J. P. C. Southall, Ed. \& Trans.). New York: Dover.

Hendrickson, A. E., Hunt, S. P., \& Wu, J.-Y. (1981). Immunocytochemical localization of glutamic acid decarboxylase in monkey striate cortex. Nature, 292, 605-607.

Hering, E. (1964). Outlines of $a$ theory of the light sense. Cambridge, MA: Harvard University Press.

Horton, J. C., \& Hubel, D. H. (1981). Regular patchy distribution of cytochrome oxidase staining in primary visual cortex of macaque monkey. Nature, 292, 762-764.

Hubel, D. H., \& Livingstone, M. S. (1981). Regions of poor orientation tuning coincide with patches of cytochrome oxidase staining in monkey striate cortex. Neuroscience Abstracts, 11th Annual Meeting, Los Angeles, 118.12.

HuBEL, D. H., \& WIESEL, T. N. (1977). Functional architecture of macaque monkey visual cortex. Proceedings of the Royal Society of London (B), 198, 1-59.

KaNIZSA, G. (1974). Contours without gradients or cognitive contours? Italian Journal of Psychology, 1, 93-113. 
KaUfman, L. (1974). Sight and mind: An introduction to visual perception. New York: Oxford University Press.

Kennedy, J. M. (1978). Illusory contours and the ends of lines. Perception, 7, 605-607.

KENNEDY, J. M. (1979). Subjective contours, contrast, and assimilation. In C. F. Nodine \& D. F. Fisher (Eds.), Perception and pictorial representation. New York: Praeger Press.

KENNEDY, J. M. (1981). Illusory brightness and the ends of petals: Change in brightness without aid of stratification or assimilation effects. Perception, 10, 583-585.

Kulikowski, J, J. (1978). Limit of single vision in stereopsis depends on contour sharpness. Nature, 275, 126-127.

LAND, E. H. (1977). The retinex theory of color vision. Scientific American, 237, 108-128.

LegGe, G. E., \& Rubin, G. S. (1981). Binocular interactions in suprathreshold contrast perception. Perception \& Psychophysics, 30, 49-61.

LEVELT, W. J. M. (1965). On binocular rivalry. Soesterberg: Institute for Perception, RVO-TNO.

Livingstone, M. S., \& Hubel, D. H. (1982). Thalamic inputs to cytochrome oxidase-rich regions in monkey visual cortex. Proceedings of the National Academy of Sciences, 79, 6098-6101.

MACH, E. (1866). Über die Wirkung der räumlichen Verteilung des Lichtreizes auf die Netzhaut. Sitzungsber. Akad. Wiss., Mathemnaturwiss. K1., Abt. 2, 52, 303-322.

O'Brien, V. (1958). Contour perception, illusion, and reality. Journal of the Optical Society of America, 48, 112-119.

PARKs, T. E. (1980). Subjective figures; Some unusual concomitant brightness effects. Perception, 9, 239-241.

PARKS, T. E., \& MARKs, W. (1983). Sharp-edged vs. diffuse illusory circles: The effects of varying luminance. Perception \& Psychophysics, 33, $172-176$.

Petry, S., Harbeck, A., Conway, J., \& Levey, J. (1983). Stimulus determinants of brightness and distinctions of subjective contours. Perception \& Psychophysics, 34, 169-174.

PrITCHARD, R. M. (1961). Stabilized images on the retina. Scientific American, 204, 72-78.

Pritchard, R. M., Heron, W., \& HebB, D. O. (1960). Visual perception approached by the method of stabilized images. Canadian Journal of Psychology, 14, 67-77.

RATLIF, F. (1965). Mach bands: Quantitative studies on neural networks in the retina. New York: Holden-Day.

Redies, C. \& Spillmann, L. (1981). The neon color effect in the Ehrenstein illusion. Perception, 10, 667-681.

Riggs, L. A., Ratliff, F., Cornsweet, J. C., \& Cornsweet, T. N (1953). The disappearance of steadily fixated visual test objects. Journal of the Optical Society of America, 43, 495-501.

SChNeIDER, W., \& ShifFrin, R. M. (1977). Controlled and automatic information processing, I: Detection, search, and attention. Psychological Review, 84, 1-66.

ToDorović, D. (1983). Brightness perception and the Craik-O-BrienCornsweet effect. Unpublished M.A. Thesis. Storrs: University of Connecticut.

vaN DEN BRink, G., \& KeEmink, C. J. (1976). Luminance gradients and edge effects. Vision Research. 16, 155-159.

van TuJu, H. F. J. M. (1975). A new visual illusion: Neonlike color spreading and complementary color induction between subjective contours. Acta Psychologica, 39, 441-445.

van Tuijl, H. F. J. M., \& DE Weert, C. M. M. (1979). Sensory conditions for the occurrence of the neon spreading illusion. Perception, $8,211-215$.

von Der Heydt, R., Peterhans, E., \& Baumgartner, G. (1984). Illusory contours and cortical neuron responses. Science, 224, 1260-1262.

Yarbus, A. L. (1967). Eye movements and vision. New York: Plenum Press.

ZEKI, S. (1983a). Colour coding in the cerebral cortex: The reaction of cells in monkey visual cortex to wavelengths and colours. Neuroscience, 9, 741-765.
ZEKI, S. (1983b). Colour coding in the cerebral cortex: The responses of wavelength-selective and colour coded cells in monkey visual cortex to changes in wavelength composition. Neuroscience, 9 , 767-791.

\section{APPENDIX A}

This appendix describes the neural network that was used to simulate feature-contour and boundary-contour interactions. The following simulations were done on one-dimensional fields of cells. The input pattern $\left(I_{1}, I_{2}, \ldots, I_{n}\right)$ is transformed into the output pattern $\left(\mathrm{z}_{1}, \mathrm{z}_{2}, \ldots, \mathrm{z}_{\mathrm{n}}\right)$ via the following equations.

\section{Feature Contours}

The input pattern $\left(I_{1}, I_{2}, \ldots, I_{n}\right)$ is transformed into feature contours via a feed-forward on-center off-surround network of cells undergoing shunting, or membrane equation, interactions. The activity, or potential $\mathrm{x}_{\mathrm{i}}$, of the $\mathrm{i}^{\text {th }}$ cell in a feature-contour pattern is

$$
\frac{d}{d t} x_{i}=-A x_{i}+\left(B-x_{i}\right) \sum_{k=1}^{n} I_{k} C_{k i}-\left(x_{i}+D\right) \sum_{k=1}^{n} I_{k} E_{k i} .
$$

Both the on-center ceofficients $C_{k i}$ and the off-surround coefficients $E_{k i}$ are Gaussian functions of intercellular distance $|k-i|$. System 1 is assumed to react more quickly than the diffusive filling-in process. Hence, we assume that each $x_{i}$ is in approximate equilibrium with respect to the input pattern. At equilibrium, $(d / d t) x_{i}=0$ and

$$
x_{i}=\frac{\sum_{k=1}^{n}\left(B C_{k i}-D E_{k i}\right) I_{k}}{A+\sum_{k=1}^{n}\left(C_{k i}+E_{k i}\right) I_{k}}
$$

The activity pattern $\left(x_{1}, x_{2}, \ldots, x_{n}\right)$ is sensitive to both the amount and the direction of contrast in edges of the input pattern (Grossberg, 1983b). These feature-contour activities generate inputs of the form

$$
F_{i}=\frac{x_{i}}{1+\alpha S_{i}}
$$

to the diffusive filling-in process. The inhibitory term $S_{i}$ is defined by the boundary-contour process in Equation 6 below.

\section{Boundary Contours}

The input pattern $\left(I_{1}, I_{2}, \ldots, I_{n}\right)$ also activates the boundarycontour process, which we represent as a feed-forward on-center off-surround network undergoing shunting interactions. This simplified view of the boundary-contour process is permissible in the present simulations because the simulations, being onedimensional and monocular, do not need to account for orientational tuning, competition, or binocular matching. Since the simulations do not probe the dynamics of illusory contour formation, the boundary completion process can also be ignored. (See Grossberg \& Mingolla, in press, for these extensions.)

As in Equation 2, the intput pattern rapidly gives rise to an activity pattern 


$$
\mathrm{y}_{\mathrm{i}}=\frac{\sum_{\mathrm{k}=1}^{\mathrm{n}}\left(\tilde{\mathrm{B}} \tilde{\mathrm{C}}_{\mathrm{ki}}-\tilde{\mathrm{D}} \tilde{\mathrm{E}}_{\mathrm{ki}}\right) \mathrm{I}_{\mathrm{k}}}{\mathrm{A}+\sum_{\mathrm{k}=1}^{\mathrm{n}}\left(\tilde{\mathrm{C}}_{\mathrm{ki}}+\tilde{\mathrm{E}}_{\mathrm{ki}}\right) \mathrm{I}_{\mathrm{k}}},
$$

where $\tilde{\mathrm{C}}_{\mathrm{ki}}$ and $\tilde{\mathrm{E}}_{\mathrm{ki}}$ are Gaussian functions of intercellular distance. It is assumed that these boundary contours are narrower than the feature contours defined by Equation 2 .

The activity pattern $\left(y_{1}, y_{2}, \ldots, y_{n}\right)$ is sensitive both to the direction and amount of contrast in the input pattern $\left(I_{1}, I_{2}, \ldots\right.$, $\left.I_{n}\right)$. The sensitivity to the direction of contrast is progressively eliminated by the following operations. Let the output signals from $B C S$ to $M B C$ that are elicited by activity $y_{i}$ equal $f\left(y_{i}\right)$, where $f(w)$ is a sigmoid signal of the rectified part of $y_{i}$; viz,

$$
\mathrm{f}\left(\mathrm{y}_{\mathrm{i}}\right)=\frac{\beta\left[\left(\mathrm{y}_{\mathrm{i}}\right)^{+}\right]^{\gamma}}{1+\delta\left[\left(\mathrm{y}_{\mathrm{i}}\right)^{+}\right]^{\gamma}} .
$$

The notation $[w]^{+}=\max (w, 0)$ and $\gamma>1$. The output signals $f\left(y_{i}\right)$ are spatially distributed before influencing cell compartments of the cell syncytium. The total signal to the $\mathrm{i}^{\text {th }}$ cell compartment due to the activity pattern $\left(y_{1}, y_{2}, \ldots, y_{n}\right)$ is

$$
S_{i}=\sum_{k=1}^{n} G_{i k} f\left(y_{k}\right)
$$

where $G_{i k}$ is a Gaussian function of intercellular distance. This Gaussian falloff is less narrow than that of boundary contours in Equation 4, but more narrow than that of feature contours in Equation 2.

\section{Diffusive Filling-In}

The activity $z_{i}$ of the $i^{\text {th }}$ cellular compartment of the cellular syncytium obeys the nonlinear diffusion equation

$$
\frac{d}{d t} z_{i}=-H z_{i}+J_{i+1, i}\left(z_{i+1}-z_{i}\right)+J_{i-1, i}\left(z_{i-1}-z_{i}\right)+F_{i}
$$

where the input $F_{i}$ is defined by Equation 3 . The diffusion coefficients $J_{i}+1, i$ and $J_{i-1, i}$ are determined by boundary contour signals according to equations of the form

$$
\mathrm{J}_{\mathrm{i}+1, \mathrm{i}}=\frac{\lambda}{1+\varkappa\left[\mathrm{S}_{\mathrm{i}+1}-\Gamma\right]^{+}+\varkappa\left[\mathrm{S}_{\mathrm{i}}-\Gamma\right]^{+}}
$$

and

$$
J_{i-1, i}=\frac{\lambda}{1+\varkappa\left[S_{i-1}-\Gamma\right]^{+}+\varkappa\left[S_{i}-\Gamma\right]^{+}},
$$

where the threshold $\Gamma>0$. Thus, an increase in the boundary signal $S_{i}$ decreases both diffusion coefficients $J_{i}+1, i$ and $J_{i-1, i}$. The feature-contour signal $F_{i}$ also decreases when the boundary signal $S_{i}$ increases. In Equations 3, 8, and 9, the inhibitory effects of boundary signals $S_{i}$ on cell compartment membranes act via shunting inhibition. A positive threshold $\Gamma$ occurs in Equations 8 and 9, but not in Equation 3, because, we assume, the intercompartmental membranes that regulate diffusion of ac- tivity between compartments are less accessible to the signals $\mathrm{S}_{\mathrm{i}}$ than are the exterior surface membranes that bound the cellular syncytium.

The following parameters were used in all the simulations with Equations 1-9. We let

$$
\begin{aligned}
& \mathrm{C}_{\mathrm{ik}}=\mathrm{C} \exp \left\{-\ln 2[(\mathrm{i}-\mathrm{k}) / \mu]^{2}\right\}, \\
& \mathrm{E}_{i \mathrm{k}}=\mathrm{E} \exp \left\{-\ln 2[(\mathrm{i}-\mathrm{k}) / \nu]^{2}\right\}, \\
& \tilde{\mathrm{C}}_{\mathrm{ik}}=\tilde{\mathrm{C}} \exp \left\{-\ln 2[(\mathrm{i}-\mathrm{k}) / \tilde{\mu}]^{2}\right\}, \\
& \tilde{\mathrm{E}}_{\mathrm{ik}}=\tilde{\mathrm{E}} \exp \left\{-\ln 2[(\mathrm{i}-\mathrm{k}) / \tilde{\nu}]^{2}\right\},
\end{aligned}
$$

and

$$
\mathrm{G}_{\mathrm{ik}}=\mathrm{G} \exp \left\{-\ln 2[(\mathrm{i}-\mathrm{k}) / \omega]^{2}\right\}
$$

where $\mathrm{A}=1, \mathrm{~B}=96, \mathrm{C}=.0625, \mathrm{E}=.0625, \mathrm{G}=.2349$, $\mathrm{H}=1, \tilde{\mathrm{A}}=1, \tilde{\mathrm{B}}=35.5546, \tilde{\mathrm{C}}=50, \tilde{\mathrm{D}}=12.5828, \tilde{\mathrm{E}}=$ $50, \beta=4 \times 10^{10}, \gamma=5, \delta=1 \times 10^{10}, \tilde{\mu}=.5, \bar{\nu}=1.5$.

The remaining parameters vary from simulation to simulation and will be given for each figure by title.

\section{(1) Two-Step and Five-Step Illusions (Figures 7 and 8)}

$$
\begin{aligned}
\mathrm{n} & =3,500, \\
\mathrm{D} & =9.12, \\
\alpha & =1, \\
\mu & =10, \\
\nu & =100, \\
\lambda & =1.926 \times 10^{6}, \\
\varkappa & =1.926 \times 10^{7}, \\
\omega & =1, \\
\Gamma & =1.7 .
\end{aligned}
$$

The inputs. The inputs consist of a step input filtered through a Gaussian kernel with a set of ramping functions superimposed on the output of the filter. The steady state level of the output is extended outward to simulate viewing the central portion of an indefinitely large field with ramps superimposed. In the twocusp pattern of Figure 7a,

$$
I_{k}=\Phi(k-350 / 100)-\Phi(k-3150 / 100)+R_{k}^{(2)},
$$

where

$$
\Phi(x)=1 / \sqrt{2 \pi} \int_{-\infty}^{x} e^{-1 / 2 z^{2}} d z
$$

and

$$
\begin{aligned}
& \mathbf{R}_{\mathrm{k}}^{(2)}= \\
& \begin{cases}0 & \text { if } 0 \leq \mathrm{k} \leq 1450 \\
.12 \tan [.9 \pi / 2(1451-\mathrm{k} / 149)] / \tan [.9 \pi /] & \text { if } 1451 \leq \mathrm{k} \leq 1600 \\
.12 \tan [.9 \pi / 2(1750.5-\mathrm{k} / 149.5)] / \tan [.9 \pi / 2] & \text { if } 1601 \leq \mathrm{k} \leq 1900 \\
.12 \tan [.9 \pi / 2(2050-\mathrm{k} / 149)] / \tan [.9 \pi / 2] & \text { if } 1901 \leq \mathrm{k} \leq 2050 \\
0 & \text { if } 2051 \leq \mathrm{k} \leq 3500 .\end{cases}
\end{aligned}
$$


In the five-cusp pattern of Figure 8a,

$$
\mathrm{I}_{\mathrm{k}}=\Phi(\mathrm{k}-350 / 100) 1 \Phi(\mathrm{k}-3150 / 100)+\mathrm{R}_{\mathrm{k}}^{(5)}
$$

where

$R_{\mathrm{k}}^{(5)}=$

$\begin{cases}0 & \text { if } 0 \leq \mathrm{k} \leq 1000 \\ .12 \tan [.9 \pi / 2(1001-\mathrm{k} / 149)] / \tan [.9 \pi / 2] & \text { if } 1001 \leq \mathrm{k} \leq 1450 \\ .12 \tan [.9 \pi / 2(1600.5-\mathrm{k} / 149.5)] / \tan [.9 \pi / 2] & \text { if } 1451 \leq \mathrm{k} \leq 1750 \\ .12 \tan [.9 \pi / 2(1900.5-\mathrm{k} / 149.5)] / \tan [.9 \pi / 2] & \text { if } 1751 \leq \mathrm{k} \leq 2050 \\ .12 \tan [.9 \pi / 2(2300.5-\mathrm{k} / 149.5)] / \tan [.9 \pi / 2] & \text { if } 2051 \leq \mathrm{k} \leq 2350 \\ .12 \tan [.9 \pi / 2(2500.5-\mathrm{k} / 149.5)] / \tan [.9 \pi / 2] & \text { if } 2351 \leq \mathrm{k} \leq 2650 \\ .12 \tan [.9 \pi / 2(2800-\mathrm{k} / 149)] / \tan [.9 \pi / 2] & \text { if } 2651 \leq \mathrm{k} \leq 2800 . \\ 0 & \text { if } 2801 \leq \mathrm{k} \leq 3500 .\end{cases}$

(3) Bergström Brightness Paradox (Figures 12 and 13)

$$
\begin{aligned}
& \mathrm{n}=700 \\
& \mathrm{D}=12, \\
& \alpha=4, \\
& \mu=10, \\
& \nu=60, \\
& \lambda=1000, \\
& \varkappa=116.7, \\
& \omega=10, \\
& \Gamma=2.6 .
\end{aligned}
$$

The inputs. The inputs represent Bergström's experimental inputs. The inputs consist of two normal curves splined together at \pm 3 standard deviations away from the 50 percentile point and placed on a pedestal. Thus, in Figure 12a,

$$
I_{k}=\left\{\begin{array}{lc}
0 & \text { if } 0 \leq k \leq 149 \\
.6+.4 \Phi(3(1-(k-149 / 100))) & \text { if } 150 \leq k \leq 349 \\
.2+.4 \Phi(3(1-(k-349 / 100))) & \text { if } 350 \leq k \leq 549 \\
0 & \text { if } 550 \leq k \leq 700 .
\end{array}\right.
$$

In Figure 13a, the inputs were chosen to be four steps of length 100 whose value is equal to the corresponding average value in the previous set of runs. Thus,

$$
I_{k}= \begin{cases}0 & \text { if } 0 \leq k \leq 150 \\ .94 & \text { if } 150 \leq k \leq 249 \\ .60 & \text { if } 250 \leq k \leq 349 \\ .54 & \text { if } 350 \leq k \leq 449 \\ .26 & \text { if } 450 \leq k \leq 549 \\ 0 & \text { if } 550 \leq k \leq 700\end{cases}
$$

(3) Hamada Brightness Paradox (Figures 15 and 16)

$$
\begin{aligned}
& \mathrm{n}=700, \\
& \mathrm{D}=14.4, \\
& \alpha=1, \\
& \mu=1,
\end{aligned}
$$

$$
\begin{aligned}
\nu & =6, \\
\lambda & =6000, \\
\varkappa & =500, \\
\omega & =1, \\
\Gamma & =1.6 .
\end{aligned}
$$

The inputs. The inputs were chosen to simulate Hamada's experimental displays. The inputs consist of a step input filtered through a Gaussian filter with a parabolic segment superimposed on the output of this filter. Specifically, in Figure 15a, let

$$
I_{k}=.3 \Phi(k-351 / 100)-.3 \Phi(k-1350 / 100)+P_{k},
$$

where

$$
P_{k}= \begin{cases}0 & \text { if } 0 \leq k \leq 750 \\ .05454(850.5-k / 99.5)^{2} & \text { if } 751 \leq k \leq 950 \\ 0 & \text { if } 951 \leq k \leq 1700\end{cases}
$$

In Figure 16a,

$$
I_{k}=.3 \Phi(k-351 / 100)-.3 \Phi(k-1350 / 100)-P_{k} .
$$

\section{APPENDIX B}

The following system of equations defines a binocular interaction capable of supporting a filling-in resonant exchange, or FIRE (Cohen \& Grossberg, 1984; Grossberg, 1983b).

\section{Monocular Representations}

Left Field:

$$
\begin{aligned}
\frac{\mathrm{d}}{\mathrm{dt}} \mathrm{x}_{\mathrm{iL}}= & -A \mathrm{x}_{\mathrm{iL}}+\left(\mathrm{B}-\mathrm{x}_{\mathrm{iL}}\right) \sum_{\mathrm{k}=1}^{\mathrm{n}} \mathrm{I}_{\mathrm{kL}}\left[\mathrm{J}_{\mathrm{kL}}+\mathrm{z}_{\mathrm{k}}\right]^{+} \mathrm{C}_{\mathrm{ki}} \\
& -\left(\mathrm{x}_{\mathrm{iL}}+\mathrm{D}\right) \sum_{\mathrm{k}=1}^{\mathrm{n}} \mathrm{I}_{\mathrm{kL}}\left[\mathrm{J}_{\mathrm{kL}}+\mathrm{z}_{\mathrm{k}}\right]^{+} \mathrm{E}_{\mathrm{ki}}
\end{aligned}
$$

and right field:

$$
\begin{aligned}
\frac{d}{d t} x_{i R}= & -A x_{i R}+\left(B-x_{i R}\right) \sum_{k=1}^{n} I_{k R}\left[J_{k R}+z_{k}\right]^{+} C_{k i} \\
& -\left(x_{i R}+D\right) \sum_{k=1}^{n} I_{k R}\left[J_{k R}+z_{k}\right]^{+} E_{k i}
\end{aligned}
$$

where $[\mathrm{w}]^{+}=\max (\mathrm{w}, 0)$.

\section{Binocular Matching (Match Field)}

$$
y_{i}=\frac{\sum_{k=1}^{n} \tilde{F}_{k i}\left[f\left(x_{k L}\right)+f\left(x_{k R}\right)\right]}{\tilde{A}+\sum_{k=1}^{n} \tilde{G}_{k i}\left[f\left(x_{k L}\right)+f\left(x_{k R}\right)\right]},
$$


where

$$
\tilde{\mathrm{F}}_{\mathbf{k i}}=\tilde{\mathrm{B}} \tilde{\mathrm{C}}_{\mathbf{k i}}-\tilde{\mathrm{D}} \tilde{\mathrm{E}}_{\mathbf{k i}}
$$

and

$$
\tilde{\mathrm{G}}_{\mathrm{ki}}=\tilde{\mathrm{C}}_{\mathrm{ki}}+\tilde{\mathrm{E}}_{\mathbf{k i}}
$$

Binocular-To-Monocular Feedback (Filtered Match Field)

$$
z_{i}=\frac{\sum_{k=1}^{n} F_{k i}^{*} g\left(y_{k}\right)}{A^{*}+\sum_{k=1}^{n} G_{k i}^{*} g\left(y_{k}\right)},
$$

where

$$
\mathrm{F}_{\mathbf{k} i}^{*}=\mathrm{B}^{*} \mathrm{C}_{\mathbf{k i}}^{*}-\mathrm{D}^{*} \mathrm{E}_{\mathbf{k}}^{*}
$$

and

$$
\mathrm{G}_{\mathrm{ki}}^{*}=\mathrm{C}_{\mathbf{k i}}^{*}+\mathrm{E}_{\mathbf{k i}}^{*}
$$

Equation 10 describes the response of the activities $x_{i L}, i=1$, $2, \ldots, n$, in the left monocular representation. Each $x_{i L}$ obeys a shunting equation in which both the excitatory interaction coefficients $C_{k i}$ and the inhibitory interaction coefficients $E_{k i}$ are Gaussian functions of the distance between $v_{k}$ and $v_{i}$. Two types of simulations have been studied:

Additive inputs. All $\mathrm{I}_{\mathrm{kL}}$ are chosen equal. The terms $\mathrm{J}_{\mathrm{kL}}$ register the input pattern and summate with the binocular-tomonocular feedback functions $z_{k}$. This is the form of the system that appears in the simulations reported herein.

Shunting inputs. All $\mathrm{J}_{\mathbf{k L}}$ are chosen equal. The terms $\mathrm{I}_{\mathbf{k L}}$ register the input pattern. The binocular-to-monocular feedback functions $\mathbf{Z}_{\mathbf{k}}$ modulate the system's sensitivity to the inputs $I_{k L}$ in the form of gain control signals.

Equation 11 , for the activities $x_{i R}, i=1,2, \ldots, n$, in the right monocular representation, has a similar interpretation. Note that the same binocular-to-monocular feedback functions $z_{k}$ are fed back to the left and right monocular representations.

The binocular matching stage (12) obeys an algebraic equation rather than a differential equation due to the simplifying assumption that the differential equation for the matching activities $y_{i}$ reacts quickly to the monocular signals $f\left(x_{k L}\right)$ and $\mathrm{f}\left(\mathrm{x}_{\mathrm{kR}}\right)$. Consequently, $\mathrm{y}_{\mathrm{i}}$ is always in an approximate equilibrium with respect to its input signals. This equilibrium equation says that the monocular inputs $f\left(x_{k L}\right)$ and $f\left(x_{k R}\right)$ are added before being matched by the shunting interaction. The signal functions $f(w)$ are chosen to be sigmoid functions of activity $w$. The excitatory interaction coefficients $\tilde{\mathrm{C}}_{\mathrm{ki}}$ and inhibitory interaction coefficients $\tilde{\mathrm{E}}_{\mathrm{ki}}$ are chosen to be Gaussian functions of distance. The spatial decay rates of $C_{k i}, \tilde{C}_{k i}$, and $C_{k i}^{*}$ are chosen equal. The spatial decay rates of $E_{k i}, \tilde{E}_{k i}$, and $E_{k i}^{*}$ are chosen equal. The on-center is chosen narrower than the off-surround.

After the monocular signal patterns $\left(f\left(x_{1 L}\right), f\left(x_{2 L}\right), \ldots, f\left(x_{n L}\right)\right)$ and $\left(\mathrm{f}\left(\mathrm{x}_{1 \mathrm{R}}\right), \mathrm{f}\left(\mathrm{x}_{2 \mathrm{R}}\right), \ldots, \mathrm{f}\left(\mathrm{x}_{\mathrm{nR}}\right)\right)$ are matched at the binocular matching stage, the binocular activities $y_{k}$ are rectified by the output signal function $g\left(y_{k}\right)$, which is typically chosen to be a sigmoid function of $y_{k}$. Then these rectified output signals are distributed back to the monocular representations via competi- tive signals (15) with the same spatial bandwidths as are used throughout the computation. The parameters used in these simulations are exhaustively listed in Cohen and Grossberg (1984).

The parameters used herein are the same as the parameters used in simulations 15-23 of Cohen and Grossberg (1984), except that in the present simulations we chose $n=200$.

Inputs

The inputs are defined in terms of the functions

$$
\mathrm{N}_{\mathrm{k}}=.025 \Phi[\sqrt{2} / 5(\mathrm{k}-15)]-.025 \Phi[\sqrt{2} / 5(\mathrm{k}-186)]
$$

Fechner's Paradox (Figure 20):

(D) Let $\mathrm{J}_{\mathrm{kL}}=\mathrm{N}_{\mathrm{k}}, 1 \leq \mathrm{k} \leq 200$, and

$$
J_{k R}= \begin{cases}N_{k} & \text { if } 1 \leq k \leq 80 \\ 0 & \text { if } 81 \leq k \leq 120 \\ N_{k} & \text { if } 121 \leq k \leq 200 .\end{cases}
$$

(E) Let $\mathrm{J}_{\mathrm{kL}}=0,1 \leq \mathrm{k} \leq 200$, and

$$
J_{k R}= \begin{cases}N_{k} & \text { if } 1 \leq k \leq 80 \\ 0 & \text { if } 81 \leq k \leq 120 \\ N_{k} & \text { if } 121 \leq k \leq 200 .\end{cases}
$$

(F) Let

$$
J_{k L}=J_{k R}= \begin{cases}N_{k} & \text { if } 1 \leq k \leq 80 \\ 0 & \text { if } 81 \leq k \leq 120 \\ N_{k} & \text { if } 121 \leq k \leq 200\end{cases}
$$

(G) Let

$$
\mathbf{J}_{\mathrm{kL}}= \begin{cases}\mathrm{N}_{\mathrm{k}} & \text { if } 1 \leq \mathrm{k} \leq 70 \\ 0 & \text { if } 71 \leq \mathrm{k} \leq 80 \\ \mathrm{~N}_{\mathrm{k}} & \text { if } 81 \leq \mathrm{k} \leq 120 \\ 0 & \text { if } 121 \leq \mathrm{k} \leq 130 \\ \mathrm{~N}_{\mathrm{k}} & \text { if } 131 \leq \mathrm{k} \leq 200\end{cases}
$$

and

$$
J_{k R}= \begin{cases}N_{k} & \text { if } 1 \leq k \leq 70 \\ 0 & \text { if } 71 \leq k \leq 130 \\ N_{k} & \text { if } 131 \leq k \leq 200 .\end{cases}
$$

Brightness Averaging and Summation (Figure 21):

(A) Let $J_{k R}=N_{k}, 1 \leq k \leq 200$, and

$$
J_{k L}= \begin{cases}N_{k} & \text { if } 1 \leq k \leq 80 \\ N_{k}+.009 & \text { if } 81 \leq k \leq 120 \\ N_{k} & \text { if } 121 \leq k \leq 200 .\end{cases}
$$

(B) Let $\mathrm{J}_{\mathrm{kR}}=\mathrm{N}, 1 \leq \mathrm{k} \leq 200$, and

$$
J_{k L}= \begin{cases}N_{k} & \text { if } 1 \leq k \leq 80 \\ 0 & \text { if } 81 \leq k \leq 120 \\ N_{k} & \text { if } 121 \leq k \leq 200 .\end{cases}
$$


(C) Let

$$
J_{k L}= \begin{cases}N_{k} & \text { if } 1 \leq k \leq 80 \\ N_{k}+.009 & \text { if } 81 \leq k \leq 120 \\ N_{k} & \text { if } 121 \leq k \leq 200\end{cases}
$$

and

$$
J_{k R}= \begin{cases}N_{k} & \text { if } 1 \leq k \leq 80 \\ 0 & \text { if } 81 \leq k \leq 120 \\ N_{k} & \text { if } 120 \leq k \leq 200 .\end{cases}
$$

(D) Let

$$
J_{k L}=J_{k R}= \begin{cases}N_{k} & \text { if } 1 \leq k \leq 80 \\ N_{k}+.009 & \text { if } 81 \leq k \leq 120 \\ N_{k} & \text { if } 121 \leq k \leq 200 .\end{cases}
$$

The input patterns in Figures 24d and 24e are listed below to illustrate the parameter choices used in this figure and to characterize the FIRE patterns depicted in Figure 25.

Ganzfeld Plus Dark Figure (Figure 25a):

Let $J_{k L}=N_{k}, 1 \leq k \leq 200$, and

$$
J_{k R}= \begin{cases}N_{k} & \text { if } 1 \leq k \leq 80 \\ 0 & \text { if } 81 \leq k \leq 120 \\ N_{k} & \text { if } 121 \leq k \leq 200 .\end{cases}
$$

Increment Plus Dark Figure (Figure 25b):

Let

$$
J_{k L}= \begin{cases}N_{k} & \text { if } 1 \leq k \leq 80 \\ N_{k}+.009 & \text { if } 81 \leq k \leq 120 \\ N_{k} & \text { if } 121 \leq k \leq 200\end{cases}
$$

and

$$
J_{k R}= \begin{cases}N_{k} & \text { if } 1 \leq k \leq 80 \\ 0 & \text { if } 81 \leq k \leq 120 \\ N_{k} & \text { if } 121 \leq k \leq 200 .\end{cases}
$$

Boundary Plus Dark Figure (Figure 26a):

Let

$$
J_{k L}= \begin{cases}N_{k} & \text { if } 1 \leq k \leq 70 \\ 0 & \text { if } 71 \leq k \leq 80 \\ N_{k} & \text { if } 81 \leq k \leq 120 \\ 0 & \text { if } 121 \leq k \leq 130 \\ N_{k} & \text { if } 131 \leq k \leq 200\end{cases}
$$

and

$$
J_{k R}=\quad \begin{cases}N_{k} & \text { if } 1 \leq k \leq 70 \\ 0 & \text { if } 71 \leq k \leq 130 \\ N_{k} & \text { if } 131 \leq k \leq 200 .\end{cases}
$$

Increment in Boundary (Figure 26b):

Let

$$
J_{k L}= \begin{cases}N_{k} & \text { if } 1 \leq k \leq 70 \\ 0 & \text { if } 71 \leq k \leq 80 \\ N_{k}+.009 & \text { if } 81 \leq k \leq 120 \\ 0 & \text { if } 121 \leq k \leq 130 \\ N_{k} & \text { if } 131 \leq k \leq 200\end{cases}
$$

and

$$
J_{k R}= \begin{cases}N_{k} & \text { if } 1 \leq k \leq 70 \\ 0 & \text { if } 71 \leq k \leq 130 \\ N_{k} & \text { if } 131 \leq k \leq 200 .\end{cases}
$$

